\title{
Wideband Channel Modeling and Intercarrier Interference Cancellation for Vehicle-to-Vehicle Communication Systems
}

\author{
Xiang Cheng, Senior Member, IEEE, Qi Yao, Miaowen Wen, Cheng-Xiang Wang, Senior Member, IEEE, \\ Ling-Yang Song, Senior Member, IEEE, and Bing-Li Jiao, Member, IEEE
}

\begin{abstract}
In this paper, we propose a new regular-shaped geometry-based stochastic model (RS-GBSM) for non-isotropic scattering wideband multiple-input multiple-output vehicle-tovehicle (V2V) Ricean fading channels. By correcting the unrealistic assumption widely used in current RS-GBSMs, the proposed model can more practically study the impact of the vehicular traffic density on channel statistics for different time delays. From the proposed model, we derive the Doppler power spectral density (PSD) and find that highly dynamic Doppler spectrum appears for V2V channels. Excellent agreement is achieved between the derived Doppler PSD and measured data, demonstrating the utility of the proposed model. To combat the intercarrier interference (ICI) caused by highly dynamic Doppler spectrum in real orthogonal frequency division multiplexing based $\mathrm{V} 2 \mathrm{~V}$ systems, this paper proposes a new type of ICI cancellation scheme, named as precoding based cancellation (PBC) scheme. The proposed scheme can be easily implemented into real V2V systems with the same ICI mitigation performance as the current best ICI cancellation scheme that has high complexity. To further improve the performance of the proposed PBC scheme, a new phase rotation aided (PRA) method, namely constant PRA (CPRA) method, is proposed. Compared with the existing PRA method, the CPRA method has better performance and much less implementation complexity. Therefore, the proposed PBC scheme with the CPRA method is the best ICI cancellation scheme for real V2V systems.
\end{abstract}

Index Terms-Vehicle-to-vehicle wideband channels, geometrybased stochastic model (GBSM), orthogonal frequency division multiplexing (OFDM), intercarrier interference (ICI) cancellation, phase rotation aided method.

Manuscript received March 1, 2012; revised July 14, 2012.

X. Cheng, M. Wen, S.-L. Yang, and B.-L. Jiao are with the School of Electronics and Computer Science, Peking University, Beijing 100871, China (e-mail: \{xiangcheng, wenmiaowen, lingyang.song, jiaobl\}@pku.edu.cn). X. Cheng is also with The State Key Laboratory of Integrated Services Networks, Xidian University, Xian, China.

Q. Yao and C.-X. Wang (corresponding author) are with the School of Information Science and Engineering, Shandong University, Jinan 250100, China, and the Joint Research Institute for Signal and Image Processing, School of Engineering \& Physical Sciences, Heriot-Watt University, Edinburgh EH14 4AS, UK (e-mail: \{q.yao, cheng-xiang.wang\}@hw.ac.uk).

This work was jointly supported by the National 973 project (Grant no. 2013CB336700), the National Natural Science Foundation of China (Grant no. 61101079), the Science Foundation for the Youth Scholar of Ministry of Education of China (Grant no. 20110001120129), the State Key Laboratory of Integrated Services Networks, Xidian University (Grant no. ISN13-05), the Ministry of Transport of China (Grant no. 2012-364-X03104), RCUK for the UK-China Science Bridges Project: R\&D on (B) $4 G$ Wireless Mobile Communications, the Key Laboratory of Cognitive Radio and Information Processing (Guilin University of Electronic Technology), the Ministry of Education, China (Grant No.: 2013KF01), and the Fundamental Research Program of Shenzhen City (Grant Nos. JCYJ20120817163755061 and JC201005250067A).

Digital Object Identifier 10.1109/JSAC.2013.SUP.0513039

\section{INTRODUCTION}

V EHICLE-TO-VEHICLE (V2V) communications play an important role in vehicular ad hoc networks and intelligent transportation systems that aim to minimize traffic accidents, improve traffic efficiency, and enable some new applications such as mobile infotainment. As a new emerging communication system, many research challenges and standardization work have to be addressed before $\mathrm{V} 2 \mathrm{~V}$ communication systems are widely developed. The first part of this paper will concentrate on one of the most important and fundamental challenges: how to properly characterize V2V channels? To practically analyze and design V2V systems, it is necessary to have reliable knowledge of the underlying propagation channel and the corresponding realistic yet easyto-use channel model.

Channel measurements for narrowband and wideband V2V channels were reported in [1][2]. As mentioned in [29], in terms of the modeling approach, V2V channel models can be categorized as geometry-based deterministic models [4], non-geometrical stochastic models [2], and geometry-based stochastic models (GBSMs) that can be further classified as regular-shaped GBSMs (RS-GBSMs) [5]-[11] and irregularshaped GBSMs [12]. To preserve the mathematical tractability, RS-GBSMs assume that all the effective scatterers are located on a regular shape (e.g., one/two-ring, ellipse, etc.). Akki and Haber [5] were the first to propose a RS-GBSM for isotropic single-input single-output (SISO) V2V Rayleigh fading channels. In [6], generic RS-GBSM was proposed with both single- and double-bounced rays for non-isotropic multiple-input multiple-output (MIMO) V2V Ricean fading channels. In [7]-[9], we proposed RS-GBSMs that for the first time have the ability to study the impact of the vehicular traffic density (VTD) on channel statistics.

However, all the above mentioned RS-GBSMs are narrowband V2V channel models. To the best of the authors' knowledge, only two wideband RS-GBSMs are available in the current literature. The first one was proposed in [11] for MIMO V2V Ricean fading channels. However, the model cannot describe the channel statistics for different time delays, which are important for $\mathrm{V} 2 \mathrm{~V}$ channels [1]. Based on the tapped delay line (TDL) structure, the second one was proposed in [10] and can investigate the channel statistics for different time delays, i.e., per-tap channel statistics. However, this model simply assumed that the moving scatterers and static scatterers 
have the same Doppler frequency. This unrealistic assumption under-estimates the dynamics of the Doppler spectrum and therefore some channel statistics derived in [10] are inaccurate. Note that this unrealistic assumption has been used in all the V2V RS-GBSMs [7]-[10] that have the ability to study the impact of the VTD on channel statistics.

Due to the obvious advantages such as high spectral efficiency and the ability to mitigate frequency-selective multipath fading and intersymbol interference, orthogonal frequency division multiplexing (OFDM) technique has been widely used in many communication systems, including IEEE 802.11p based V2V communication systems [13]. The performance of OFDM systems is determined by the specific orthogonal properties among OFDM subcarriers. The subcarriers' orthogonality can be easily destroyed by carrier frequency offset (CFO), leading to intercarrier interference (ICI). ICI problem becomes more serious for $\mathrm{V} 2 \mathrm{~V}$ communication systems because of the high Doppler frequency caused by the fast movement of the transmitter (Tx), receiver (Rx), and scatterers. The second part of this paper will focus on the fundamental issue on the proper use of OFDM technique in V2V systems: how to simply and effectively mitigate ICI? To deal with the ICI problem, several approaches have been proposed [14]-[26]. Among them, the ICI self-cancellation scheme [17]-[22] and two-path cancellation scheme [23][24] have been widely accepted due to their simple and effective ICI mitigation capability.

ICI self-cancellation scheme repeats OFDM symbols with special mapping operations in one OFDM block. ICI selfcancellation schemes cannot remain their theoretical ICI mitigation performance when they are used in real V2V systems. This is the main drawback of the implementation of ICI self-cancellation schemes into real V2V systems. Unlike ICI self-cancellation schemes, two-path cancellation schemes transmit the data copies in two concatenated OFDM blocks, which are referred to as two independent paths. So far the most promising two-path cancellation schemes are parallel cancellation (PC) scheme [23] and conjugate cancellation (CC) scheme [24]. The bottleneck of the implementation of two-path cancellation schemes into real V2V systems is the high realization complexity due to its two separate OFDM transceivers.

More recently, to further improve the performance of the CC scheme, the authors in [25] added an artificial phase rotation into the $\mathrm{CC}$ scheme to propose a new two-path cancellation method, namely phase rotated conjugate cancellation (PRCC) scheme. The phase rotation aided (PRA) method can be used for any ICI self-cancellation and two-path cancellation schemes to further improve their ICI mitigation performance. However, to obtain the accurate phase rotation at the Tx in real OFDM systems, one needs an extra powerful estimator and a feedback link, which increase the system complexity and reduce the real-time system performance. Moreover, the powerful estimator could have estimation errors that will degrade the performance of the PRA method in real applications. In addition, the PRA method is designed based on a singlevalued frequency offset and thus is not suitable for real V2V systems, having highly dynamic Doppler spectrum.

Motivated by the aforementioned gap, this paper will propose a new wideband V2V RS-GBSM and a new type of ICI cancellation scheme with a new PRA method for real V2V systems. The main contributions and novelties of this paper are listed as follows.

1) We propose a new wideband V2V RS-GBSM that corrects the unrealistic assumption widely used in current V2V RS-GBSMs [7]-[10] by properly describing different Doppler frequencies for moving scatterers representing moving cars and static scatterers representing static roadside objects. Therefore, the proposed model is the first RS-GBSM being able to practically investigate the impact of VTD on channel statistics. From the proposed model, we further derive the Doppler power spectral density (PSD). The derived per-tap Doppler PSDs and measurement data in [1] are compared. Excellent agreement between them demonstrates the utility of the proposed model.

2) Based on our previous observation on the relationship between the ICI self-cancellation scheme and two-path cancellation scheme [26], we propose a new type of ICI cancellation scheme, namely, precoding based cancellation $(\mathrm{PBC})$ scheme. By taking the advantages of both the ICI self-cancellation scheme and two-path cancellation scheme, the proposed PBC scheme is the best ICI cancellation scheme for real V2V systems.

3) A new PRA method is proposed and named as constant phase rotation aided (CPRA) method. Instead of using a varying phase rotation at the Tx designed based on a single-valued frequency offset, which is obtained from the powerful estimator and feedback link, the proposed CPRA method uses a constant phase rotation derived from a given frequency offset range beforehand. In real V2V systems, the CPRA method shows better ICI mitigation performance than the PRA method. More importantly, the implementation of the CPRA method is extremely simple.

\section{A New Wideband MIMO V2V RS-GBSM}

Let us now consider a wideband MIMO V2V communication system with $M_{T}$ transmit and $M_{R}$ receive omnidirectional antenna elements. Both the Tx and Rx are moving with velocities represented by the vector $\bar{v}_{S T}$ and vector $\bar{v}_{S R}$, respectively, and equipped with low elevation antennas. The proposed RS-GBSM is illustrated in Fig. 1, which is the combination of a two-ring model and a multiple confocal ellipses model incorporating LoS, single- and double-bounced rays. The two-ring model defines two rings of effective scatterers, one around the Tx and the other around the Rx. The multiple confocal ellipses model with the Tx and Rx located at the foci defines multiple ellipses of effective scatterers and represents the TDL structure. To make the proposed model able to investigate the impact of the VTD on channel statistics, the two-ring model is used to present moving scatterers, e.g., moving cars around the Tx and Rx, and the multiple confocal ellipses model is applied to present static scatterers, e.g., static roadside environment. Unlike previous RS-GBSMs, the proposed model actually considers the movement of the moving scatterers by defining the vector $\bar{v}_{S T}$ and vector $\bar{v}_{S R}$ to represent the velocity of moving scatterers around the $\mathrm{Tx}$ 


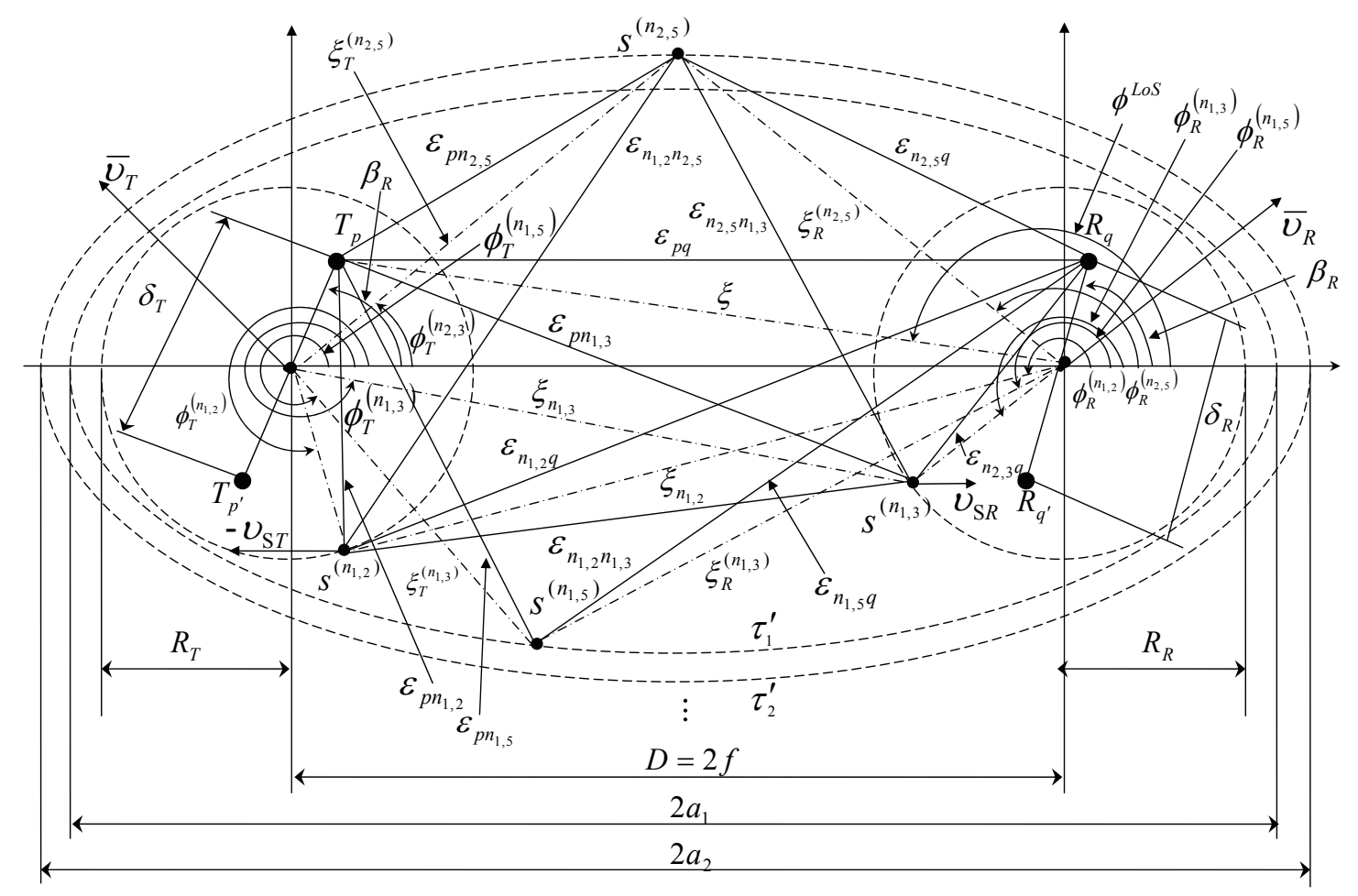

Fig. 1. A RS-GBSM combining a two-ring model and a multiple confocal ellipses model with LoS, single- and double-bounced rays for a wideband MIMO V2V channel.

and $\mathrm{Rx}$, respectively. By considering a real $\mathrm{V} 2 \mathrm{~V}$ communication scenario, where the $\mathrm{Tx}$, the $\mathrm{Rx}$, and other moving cars should move on the same road with either the same or opposite direction, their movement directions are either along the $x$-axis, i.e., $\bar{v}_{T}=v_{T}, \bar{v}_{R}=v_{R}, \bar{v}_{S T}=v_{S T_{1}}$, and $\bar{v}_{S R}=v_{S R_{1}}$, or along the opposite direction of the $x$-axis, i.e., $\bar{v}_{T}=-v_{T}, \bar{v}_{R}=-v_{R}, \bar{v}_{S T}=-v_{S T_{2}}$, and $\bar{v}_{S R}=-v_{S R_{2}}$. Therefore, for the more practical investigation of the impact of the VTD, we further distinguish the moving scatterers as the positive moving scatterers with velocities $v_{S T_{1}}$ or $v_{S R_{1}}$ and the negative moving scatterers with velocities $-v_{S T_{2}}$ or $-v_{S R_{2}}$. Suppose there are $N_{1,1}$ positive moving scatterers and $N_{1,2}$ negative moving scatterers around the Tx and $N_{1,3}$ positive moving scatterers and $N_{1,4}$ negative moving scatterers around the Rx. Similarly, suppose $N_{l, 5}$ static scatterers on the $l$ th ellipse (i.e., $l$ th tap), where $l=1,2, \ldots, L$ with $L$ being the total number of ellipses or taps. Note that the $n_{1, i}$ th $(i=1,2, \ldots, 5)$ effective scatterer is denoted by $s^{\left(n_{1, i}\right)}$ in Fig. 1. As the rays related to positive moving scatterers and negative moving scatterers in one ring have the same geometry, only the rays related to $s^{\left(n_{1,2}\right)}$ and $s^{\left(n_{1,3}\right)}$ are shown in Fig. 1. The parameters in Fig. 1 are defined in Table I.

The MIMO fading channel can be described by a matrix $\mathbf{H}(t)=\left[h_{p q}\left(t, \tau^{\prime}\right)\right]_{M_{R} \times M_{T}}$ of size $M_{R} \times M_{T}$. According to the TDL concept, the complex impulse response between the $p$ th $\left(p=1, \ldots, M_{T}\right) \mathrm{Tx}, T_{p}$, and the $q$ th $\left(q=1, \ldots, M_{R}\right) \mathrm{Rx}$, $R_{q}$, can be expressed as $h_{p q}\left(t, \tau^{\prime}\right)=\sum_{l=1}^{L} c_{l} h_{l, p q}(t) \delta\left(\tau^{\prime}-\tau_{l}^{\prime}\right)$

where $c_{l}$ represents the gain of the $l$ th tap, $h_{l, p q}(t)$ and $\tau_{l}^{\prime}$ denote the complex time-variant tap coefficient and the discrete propagation delay of the $l$ th tap, respectively. From the above RS-GBSM and applying the sum-of-sinusoids channel modeling approach [27]-[30], the complex tap coefficient for the first tap of the $T_{p}-R_{q}$ link is a superposition of the LoS, single- and double-bounced components, and can be expressed as

$$
h_{1, p q}(t)=h_{1, p q}^{L o S}(t)+\sum_{i_{1}=1}^{I_{1}} h_{1, p q}^{S B_{i_{1}}}(t)+\sum_{i_{2}=1}^{I_{2}} h_{1, p q}^{D B_{i_{2}}}(t)
$$

with

$$
\begin{aligned}
& h_{1, p q}^{L o S}(t)=\sqrt{\frac{K_{p q}}{K_{p q}+1}} e^{-j 2 \pi f_{c} \tau_{p q}} \\
& \times e^{j\left[2 \pi \frac{v_{T}}{c} f_{c} t \cos \left(\pi-\phi^{L o S}+\gamma_{T}\right)+2 \pi \frac{v_{R}}{c} f_{c} t \cos \left(\phi^{L o S}-\gamma_{R}\right)\right]} \\
& h_{1, p q}^{S B_{i_{1}}}(t)=\sqrt{\frac{\eta_{S B_{1, i_{1}}}}{K_{p q}+1}} \sum_{n_{1, i_{1}}=1}^{N_{1, i_{1}}} \frac{1}{\sqrt{N_{1, i_{1}}}} e^{j\left(\psi_{n_{1, i_{1}}}-2 \pi f_{c} \tau_{p q, n_{1, i_{1}}}\right)} \\
& \times e^{\frac{j 2 \pi f_{c} t}{c}\left(\bar{v}_{i_{1}}^{T} \cos \phi_{T}^{\left(n_{1, i_{1}}\right)}+\bar{v}_{i_{1}}^{R} \cos \phi_{R}^{\left(n_{1, i_{1}}\right)}\right)} \\
& h_{1, p q}^{D B_{i_{2}}}(t)=\sqrt{\frac{\eta_{D B_{1, i_{2}}}}{K_{p q}+1}} \sum_{n_{1, \sigma_{i_{2}}}, n_{1, \varsigma_{i_{2}}}=1}^{N_{1, \sigma_{i_{2}}}, N_{1, \varsigma_{i_{2}}}} \frac{1}{\sqrt{N_{1, \sigma_{i_{2}}} N_{1, \varsigma_{i_{2}}}}} \\
& \times e^{j\left(\psi_{n_{1, \sigma_{i}}, n_{1, \varsigma_{i_{2}}}}-2 \pi f_{c} \tau_{p q, n_{1, \sigma_{i}}, n_{1, \varsigma_{i}}}\right)} e^{\frac{j 2 \pi f_{c} t}{c} \bar{v}_{\sigma_{i_{2}}}^{T} \cos \phi_{T}^{\left(n_{1, \sigma_{i_{2}}}\right)}}
\end{aligned}
$$


TABLE I

DEFINITION OF PARAMETERS IN FIG. 1.

\begin{tabular}{|c|c|}
\hline$D$ & distance between the Tx and $\mathrm{Rx}$ \\
\hline$R_{T}, R_{T}$ & radii of the ring around the $\mathrm{Tx}$ and $\mathrm{Rx}$, respectively \\
\hline$f$ & $\begin{array}{l}\text { half length of the distance between } \\
\text { the two focal points of ellipses }\end{array}$ \\
\hline$a_{l}, b_{l}$ & $\begin{array}{l}\text { semi-major axis and semi-minor axis } \\
\text { of the } l \text { th ellipse, respectively }\end{array}$ \\
\hline $\bar{v}_{T}, \bar{v}_{R}$ & $\begin{array}{l}\text { velocities of the Tx and Rx, respectively, } \\
\text { including a magnitude and a direction }\end{array}$ \\
\hline $\bar{v}_{S T}, \bar{v}_{S R}$ & $\begin{array}{l}\text { velocities of moving scatterers in the ring around the } \mathrm{Tx} \\
\text { and } \mathrm{Rx} \text {, respectively, including a magnitude and a direction }\end{array}$ \\
\hline$\delta_{T}, \delta_{R}$ & antenna element spacings of the $\mathrm{Tx}$ and $\mathrm{Rx}$, respectively \\
\hline$\overline{\beta_{T}, \beta_{R}}$ & $\begin{array}{l}\text { orientations of the Tx and } \mathrm{Rx} \text { antenna arrays in the } \\
\mathrm{x}-\mathrm{y} \text { plane (relative to the } \mathrm{x} \text {-axis), respectively }\end{array}$ \\
\hline $\begin{array}{c}\phi_{R}^{\left(n_{1,1}\right)}, \phi_{R}^{\left(n_{1,2}\right)} \\
\phi_{R}^{\left(n_{l, 3}\right)}\end{array}$ & $\begin{array}{l}\text { AoAs of the wave traveling from an effective scatterer } \\
s^{\left(n_{1,1}\right)}, s^{\left(n_{1,2}\right)} \text {, and } s^{\left(n_{l, 3}\right)} \text { toward the Rx, respectively }\end{array}$ \\
\hline $\begin{array}{c}\phi_{T}^{\left(n_{1,1}\right)}, \phi_{T}^{\left(n_{1,2}\right)} \\
\phi_{T}^{\left(n_{l, 3}\right)}\end{array}$ & $\begin{array}{l}\text { AoDs of the wave that impinges on the effective } \\
\text { scatterer } s^{\left(n_{1,1}\right)}, s^{\left(n_{1,2}\right)} \text {, and } s^{\left(n_{l, 3}\right)} \text {, respectively. }\end{array}$ \\
\hline $\begin{array}{l}\xi, \xi_{n_{1}, 1}, \xi_{n_{1,2}} \\
\xi_{T}^{\left(n_{1,3}\right)}, \xi_{R}^{\left(n_{1}, 3\right)} \\
\xi_{T}^{\left(n_{2,3}\right)}, \xi_{R}^{\left(n_{2,3}\right)}\end{array}$ & $\begin{array}{l}\text { distances } d\left(T_{p}, R x\right), d\left(S^{\left(n_{1}, 1\right)}, R x\right), d\left(T x, S^{\left(n_{1,2}\right)}\right) \\
d\left(T x, S^{\left(n_{1}, 3\right)}\right), d\left(S^{\left(n_{1,3}\right)}, R x\right), d\left(T x, S^{\left(n_{2,3}\right)}\right) \\
\text { and } d\left(S^{\left(n_{2,3}\right)}, R x\right) \text {, respectively }\end{array}$ \\
\hline $\begin{array}{c}\varepsilon_{p n_{1, g}}, \varepsilon_{n_{1, g} q}, \varepsilon_{p n_{2,3}} \\
\varepsilon_{n_{2,3} q}, \varepsilon_{n_{1,1} n_{1,2}} \\
\varepsilon_{n_{1,1} n_{2,3}, \varepsilon_{n_{2,3} n_{1,2}}} \\
\quad(g=1,2,3) \\
\end{array}$ & $\begin{array}{l}\text { distances } d\left(T_{p}, S^{\left(n_{1}, g\right)}\right), d\left(S^{\left(n_{1, g}\right)}, R_{q}\right) \\
d\left(T_{p}, S^{\left(n_{2,3}\right)}\right), d\left(S^{\left(n_{2,3}\right)}, R_{q}\right) \\
d\left(S^{\left(n_{1}, 1\right)}, S^{\left(n_{1,2}\right)}\right), d\left(S^{\left(n_{1}\right)}, S^{\left(n_{2,3}\right)}\right) \\
\text { and } d\left(S^{\left(n_{2,3}\right)}, S^{\left(n_{1,2}\right)}\right), \text { respectively }(g=1,2,3)\end{array}$ \\
\hline
\end{tabular}

$$
\times e^{\frac{j 2 \pi f_{c} t}{c}\left(\bar{v}_{\sigma_{i_{2}}, \varsigma_{i_{2}}}^{S} \cos \phi_{S}^{\left(n_{1}, \sigma_{i_{2}}{ }^{\left.n_{1}, \varsigma_{i_{2}}\right)}\right.}+\bar{v}_{\varsigma_{i_{2}}}^{R} \cos \phi_{R}^{\left(n_{1, \varsigma_{i_{2}}}\right)}\right)}
$$

where $I_{1}=5, I_{2}=4, \sigma_{1}=\sigma_{2}=1, \sigma_{3}=\sigma_{4}=2, \varsigma_{1}=\varsigma_{3}=$ $3, \varsigma_{2}=\varsigma_{4}=4, \tau_{p q}=\varepsilon_{p q} / c, \tau_{p q, n_{1, i_{1}}}=\left(\varepsilon_{p n_{1, i_{1}}}+\varepsilon_{n_{1, i_{1} q}}\right) / c$, $\tau_{p q, n_{1, \sigma_{i}}, n_{1, \varsigma_{i}}}=\left(\varepsilon_{p n_{1, \sigma_{i}}}+\varepsilon_{n_{1, \sigma_{i_{2}}} n_{1, \varsigma_{i_{2}}}}+\varepsilon_{n_{1, \varsigma_{i_{2}}} q}\right) / c$, and $\gamma_{T}$ and $\gamma_{R}$ are the moving directions of the $\mathrm{Tx}$ and $\mathrm{Rx}$, respectively, with the value being either 0 or $\pi$, i.e., $\gamma_{T(R)}=0$ or $\pi$. The parameters $\bar{v}_{1}^{T(R)}=v_{T(R)} \cos \gamma_{T(R)}-v_{S T_{1}}, \bar{v}_{3}^{T(R)}=$ $v_{T(R)} \cos \gamma_{T(R)}-v_{S R_{1}}, \bar{v}_{2}^{T(R)}=v_{T(R)} \cos \gamma_{T(R)}+v_{S T_{2}}$, $\bar{v}_{4}^{T(R)}=v_{T(R)} \cos \gamma_{T(R)}+v_{S R_{2}}, \bar{v}_{5}^{T(R)}=v_{T(R)} \cos \gamma_{T(R)}$, $\bar{v}_{1,3(4)}^{S}=v_{S T_{1}} \mp v_{S R_{1(2)}}$, and $\bar{v}_{2,3(4)}^{S}=-v_{S T_{2}} \mp v_{S R_{1(2)}}$. The symbols $c$ and $K_{p q}$ designate the speed of light and the Ricean factor, respectively. The complex tap coefficient for other taps $\left(l^{\prime}>1\right)$ of the $T_{p}-R_{q}$ link is a superposition of the singleand double-bounced components, and can be expressed as

$$
h_{l^{\prime}, p q}(t)=h_{l^{\prime}, p q}^{S B_{5}}(t)+\sum_{i_{2}=1}^{I_{2}} h_{l^{\prime}, p q}^{D B_{i_{2}}}(t)
$$

with

$$
\begin{aligned}
& h_{l^{\prime}, p q}^{S B_{5}}(t)=\sqrt{\eta_{S B_{l^{\prime}, 5}}} \sum_{n_{l^{\prime}, 5}=1}^{N_{l^{\prime}, 5}} \frac{1}{\sqrt{N_{l^{\prime}, 5}}} e^{j\left(\psi_{n_{l^{\prime}, 5}}-2 \pi f_{c} \tau_{p q, n_{l^{\prime}, 5}}\right)} \\
& \times e^{\frac{j 2 \pi f_{c} t}{c}\left(\bar{v}_{5}^{T} \cos \phi_{T}^{\left(n_{l^{\prime}, 5}\right)}+\bar{v}_{5}^{R} \cos \phi_{R}^{\left(n_{l^{\prime}, 5}\right)}\right)}
\end{aligned}
$$

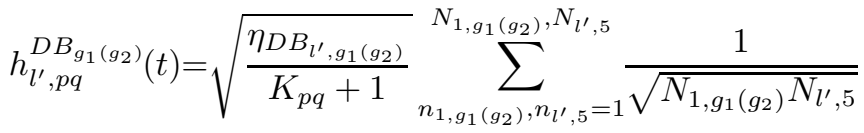

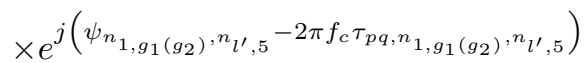

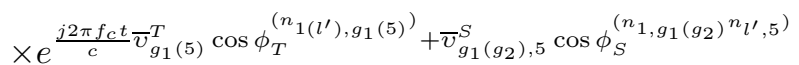

$$
\begin{aligned}
& \times e^{\frac{j 2 \pi f_{c} t}{c}\left(\bar{v}_{5\left(g_{2}\right)}^{R} \cos \phi_{R}^{\left(n l^{\prime}(1), 5\left(g_{2}\right)\right.}\right)}
\end{aligned}
$$

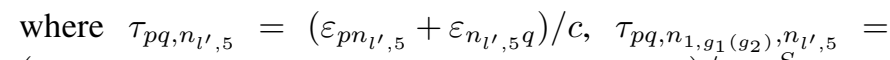
$\left(\varepsilon_{p n_{1\left(l^{\prime}\right), g_{1}(5)}}+\varepsilon_{n_{1\left(l^{\prime}\right), g_{1}(5)} n_{l^{\prime}(1), 5\left(g_{2}\right)}}+\varepsilon_{n_{l^{\prime}(1), 5\left(g_{2}\right)} q}\right) / c, \bar{v}_{1(3), 5}^{S}=$ $v_{S T_{1}\left(R_{1}\right)}, \bar{v}_{2(4), 5}^{S}=-v_{S T_{2}\left(R_{2}\right)}, g_{1}=1,2$, and $g_{2}=$ 3, 4. Energy-related parameters $\eta_{S B_{1, i_{1}}}, \eta_{D B_{1, i_{2}}}$ and $\eta_{S B_{l^{\prime}, 5}}$, $\eta_{D B_{l^{\prime}, i_{3}}}$ specify how much the single-, double-bounced rays contribute to the total scattered power of the first tap and other taps, respectively. Note that these energy-related parameters satisfy $\sum_{i_{1}=1}^{I_{1}} \eta_{S B_{1, i_{1}}}+\sum_{i_{2}=1}^{I_{2}} \eta_{D B_{1, i_{2}}}=1$ and $\eta_{S B_{l^{\prime}, 5}}+\sum_{i_{3}=1}^{I_{2}} \eta_{D B_{l^{\prime}, i_{3}}}=1$. The phases $\psi_{n_{1, i_{1}}}, \psi_{n_{1, \sigma_{i_{2}}}, n_{1, \varsigma_{i_{2}}}}$, $\psi_{n_{l^{\prime}, 5}}$, and $\psi_{n_{1, g_{1}\left(g_{2}\right)}, n_{l^{\prime}, 5}}$ are independent and identically distributed (i.i.d.) random variables with uniform distributions over $[-\pi, \pi)$.

As presented in [2], the VTD (either high or low VTD) significantly affects statistical properties at all taps of a wideband V2V channel. By using the two-ring model with the proper definition of moving velocity to mimic the moving cars and the multiple confocal ellipses model to depict the stationary roadside environment, the proposed RS-GBSM can practically investigate the impact of the VTD on channel statistics. Considering the real $\mathrm{V} 2 \mathrm{~V}$ communication environments and the different contributions of single- and double-bounced rays for $\mathrm{V} 2 \mathrm{~V}$ channel statistics, we design different taps of our model as following.

For the first tap, the single-bounced rays are generated from the scatterers located on either of the two rings or the first ellipse, while the double-bounced rays are produced from the scatterers located on both rings. This means that the first tap contains a LoS component, a two-ring model with single- and double-bounced rays, and an ellipse model with single-bounced rays, as shown in Fig. 2. For a low VTD (LVTD), the value of $K_{p q}$ is large since the LoS component can bear a significant amount of power. Also, the received scattered power is mainly from waves reflected by the static scatterers located on the first ellipse. The moving scatterers 


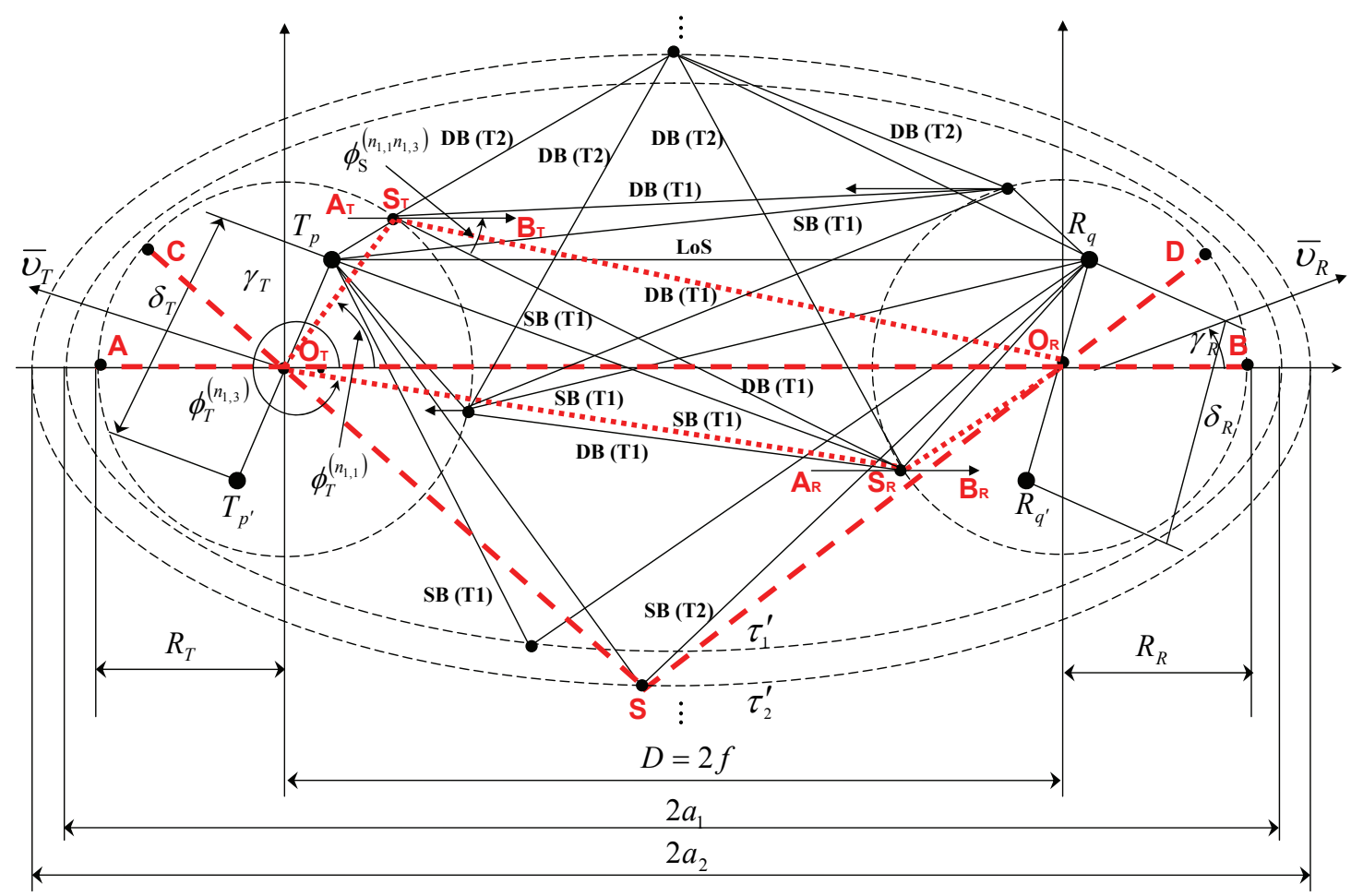

Fig. 2. Geometrical description of the LoS, single- and double-bounced rays for different taps in the proposed wideband MIMO V2V RS-GBSM. SB: single-bounce; DB: double-bounce; T1: tap 1; T2: tap 2.

located on the two rings are sparse and thus more likely to be single-bounced, rather than double-bounced. This indicates that $\eta_{S B_{1,5}}>\max \left\{\eta_{S B_{1, i}}\right\}_{i=1}^{4}>\max \left\{\eta_{D B_{1, i}}\right\}_{i=1}^{4}$. For a high VTD (HVTD), the value of $K_{p q}$ is smaller than the one in the LVTD scenario. Also, due to the large number of moving cars, the double-bounced rays of the two-ring model bear more energy than single-bounced rays of the two-ring and ellipse models, i.e, $\min \left\{\eta_{D B_{1, i}}\right\}_{i=1}^{4}>\max \left\{\eta_{S B_{1, i}}\right\}_{i=1}^{5}$. In addition, for the scenario where more cars move along the $x$-axis, we have $\left\{\eta_{S B_{1,1}}, \eta_{S B_{1,3}}\right\}>\left\{\eta_{S B_{1,2}}, \eta_{S B_{1,4}}\right\}$ and $\eta_{D B_{1,1}}>\left\{\eta_{D B_{1,2}}, \eta_{D B_{1,3}}\right\}>\eta_{D B_{1,4}}$. Similarly, for the scenario where more cars move along the opposite direction of the $x$-axis, we have $\left\{\eta_{S B_{1,2}}, \eta_{S B_{1,4}}\right\}>\left\{\eta_{S B_{1,1}}, \eta_{S B_{1,3}}\right\}$ and $\eta_{D B_{1,4}}>\left\{\eta_{D B_{1,2}}, \eta_{D B_{1,3}}\right\}>\eta_{D B_{1,1}}$.

For other taps, we assume that the single-bounced rays are generated only from the static scatterers located on the corresponding ellipse, while the double-bounced rays are caused by the scatterers from the combined one ring (either of the two rings) and the corresponding ellipse, as illustrated in Fig. 2. Note that according to the TDL structure, the double-bounced rays in one tap must be smaller in distance than the single-bounced rays on the next ellipse. As shown in Appendix $A$, this is valid only if the condition $\max \left\{R_{T}, R_{R}\right\}<\min \left\{a_{l}-a_{l-1}\right\}$ is fulfilled. For many current $\mathrm{V} 2 \mathrm{~V}$ channel measurement campaigns, e.g., in [1][2], the resolution in delay is $100 \mathrm{~ns}$. Then, the above condition can be modified as $\max \left\{R_{T}, R_{R}\right\} \leq 15 \mathrm{~m}$ by calculating the equality $2\left(a_{l}-a_{l-1}\right)=c \cdot \tau^{\prime}$ with $c=3 \times 10^{8} \mathrm{~m} / \mathrm{s}$ and $\tau^{\prime}=100 \mathrm{~ns}$. This indicates that the maximum acceptable width of the road is $30 \mathrm{~m}$, which is sufficiently large to cover most roads in reality. In other words, the proposed wideband model with the specified TDL structure is valid for different scenarios. For a LVTD, the received scattered power is mainly from waves reflected by the static scatterers located on the ellipse. This indicates that $\eta_{S B_{l^{\prime}, 5}}>\max \left\{\eta_{D B_{l^{\prime}, i}}\right\}_{i=1}^{4}$. For a HVTD, due to the large number of moving cars, the double-bounced rays from the combined one-ring and ellipse models bear more energy than the single-bounced rays of the ellipse model, i.e, $\min \left\{\eta_{D B_{l^{\prime}, i}}\right\}_{i=1}^{4}>\eta_{S B_{l^{\prime}, 5}}$. Moreover, for the scenario where more cars move along the $x$-axis, we have $\left\{\eta_{D B_{l^{\prime}, 1}}, \eta_{D B_{l^{\prime}, 3}}\right\}>\left\{\eta_{D B_{l^{\prime}, 2}}, \eta_{D B_{l^{\prime}, 4}}\right\}$. Similarly, for the scenario where more cars move along the opposite direction of the $x$-axis, we have $\left\{\eta_{D B_{l^{\prime}, 2}}, \eta_{D B_{l^{\prime}, 4}}\right\}>\left\{\eta_{D B_{l^{\prime}, 1}}, \eta_{D B_{l^{\prime}, 3}}\right\}$.

From Fig. 1, based on the application of the law of cosines in triangles and the following assumptions $\min \left\{R_{T}, R_{R}, a-\right.$ $f\} \gg \max \left\{\delta_{T}, \delta_{R}\right\}$ and $D \gg \max \left\{R_{T}, R_{R}\right\}$, and using the approximation $\sqrt{1+x} \approx 1+x / 2$ for small $x$, we have

$$
\begin{aligned}
& \varepsilon_{p q} \approx D-k_{p} \delta_{T} \cos \beta_{T}-k_{q} \delta_{R} \cos \left(\phi^{L o S}-\beta_{R}\right) \\
& \varepsilon_{p(q) n_{1, g_{1}\left(g_{2}\right)}} \approx \\
& R_{T(R)}-k_{p(q)} \delta_{T(R)} \cos \left(\phi_{T(R)}^{\left(n_{1, g_{1}\left(g_{2}\right)}\right)}-\beta_{T(R)}\right)
\end{aligned}
$$

$\varepsilon_{n_{1, g_{1}\left(g_{2}\right)} q(p)} \approx$ 


$$
\begin{aligned}
& D \mp R_{T(R)} \cos \phi_{T(R)}^{\left(n_{1, g_{1}\left(g_{2}\right)}\right)}-k_{q(p)} \delta_{R(T)} \cos \left(\phi_{R(T)}^{\left(n_{1, g_{1}\left(g_{2}\right)}\right)}-\beta_{R(T)}\right) \\
& \varepsilon_{n_{1,1} n_{1,3(4)}}=\varepsilon_{n_{1,2} n_{1,3(4)}} \approx \\
& D-R_{T} \cos \phi_{T}^{\left(n_{1,1}\right)}-R_{R} \cos \left(\phi_{R}^{\left(n_{1,1}\right)}-\phi_{R}^{\left(n_{1,2}\right)}\right) \\
& \varepsilon_{n_{1, g_{1}\left(g_{2}\right)} n_{l^{\prime}, 5}}= \\
& \sqrt{\left(\xi_{T(R)}^{\left(l^{\prime}, 5\right)}\right)^{2}+R_{T(R)}^{2}-2 \xi_{T(R)}^{\left(l^{\prime}, 5\right)} R_{T(R)} \cos \left(\phi_{T(R)}^{\left(n_{1, g_{1}\left(g_{2}\right)}\right)} \phi_{T(R)}^{\left(n_{l^{\prime}, 5}\right)}\right)} \\
& \varepsilon_{p(q) n_{l, 5}} \approx \xi_{T(R)}^{(l, 5)}-k_{p(q)} \delta_{T(R)} \cos \left(\phi_{T(R)}^{\left(n_{l, 5}\right)}-\beta_{T(R)}\right)
\end{aligned}
$$

where $\phi^{L o S} \approx \pi, k_{p}=\left(M_{T}-2 p+1\right) / 2, k_{q}=\left(M_{R}-2 q+1\right) / 2$, $\xi_{T}^{\left(n_{l, 5}\right)}=\left(a_{l}^{2}+f^{2}+2 a_{l} f \cos \phi_{R}^{\left(n_{l, 5}\right)}\right) /\left(a_{l}+f \cos \phi_{R}^{\left(n_{l, 5}\right)}\right)$, and $\xi_{R}^{\left(n_{l, 5}\right)}=b_{l}^{2} /\left(a_{l}+f \cos \phi_{R}^{\left(n_{l, 5}\right)}\right)$. Note that the AoD $\left\{\phi_{T}^{\left(n_{1, i}\right)}\right\}_{i=1}^{4}, \phi_{T}^{\left(n_{l^{\prime}, 5}\right)}$ and AoA $\left\{\phi_{R}^{\left(n_{1, i}\right)}\right\}_{i=1}^{4}, \phi_{R}^{\left(n_{l^{\prime}, 5}\right)}$ are independent for double-bounced rays, while they are interdependent for single-bounced rays. By using the results in [8][31], we can express the relationships between the AoD and AoA for the single-bounced two-ring model as $\phi_{R}^{\left(n_{1,1(2)}\right)} \approx$ $\pi-\Delta_{T} \sin \phi_{T}^{\left(n_{1,1(2)}\right)}$ and $\phi_{T}^{\left(n_{1,3(4)}\right)} \approx \Delta_{R} \sin \phi_{R}^{\left(n_{1,3(4)}\right)}$ with $\Delta_{T} \approx R_{T} / D$ and $\Delta_{R} \approx R_{R} / D$ and for the multiple confocal ellipses model as $\phi_{T}^{\left(n_{l, 5}\right)}=\arcsin \left[b_{l}^{2} \sin \phi_{R}^{\left(n_{l, 5}\right)} /\left(a_{l}^{2}+f^{2}+\right.\right.$ $\left.\left.2 a_{l} f \cos \phi_{R}^{\left(n_{l, 5}\right)}\right)\right]$. Another important angles for the proposed model are expressed as

$$
\begin{aligned}
& \phi_{S}^{\left(n_{1, \sigma_{i}} n_{1, \varsigma_{i}}\right)}= \\
& \arcsin \left[R_{T} \sin \left(\phi_{T}^{\left(n_{1, \varsigma_{i}}\right)}-\phi_{T}^{\left(n_{1, \sigma_{i_{2}}}\right)}\right) / d_{i_{2}}\right]-\phi_{T}^{\left(n_{1, \varsigma_{i}}\right)} \\
& \phi_{S}^{\left(n_{1, g_{1}\left(g_{2}\right)} n_{l^{\prime}, 5}\right)}= \\
& \arcsin \left[R_{T(R)} \sin \left(\phi_{T(R)}^{\left(n_{l^{\prime}, 5}\right)}-\phi_{T(R)}^{\left(n_{1, g_{1}\left(g_{2}\right)}\right)}\right) / d_{5(6)}\right]-\phi_{T(R)}^{\left(n_{l^{\prime}, 5}\right)}
\end{aligned}
$$

where $d_{i_{2}}=\left[R_{T}^{2}+\xi_{T}^{2}-2 R_{T} \xi_{T} \cos \left(\phi_{T}^{\left(n_{1, \varsigma_{i}}\right)}-\phi_{T}^{\left(n_{1, \sigma_{i_{2}}}\right)}\right)\right]^{1 / 2}$ with $\xi_{T} \approx D+R_{R} \cos \phi_{R}^{\left(n_{1,3(4)}\right)}$ and $d_{5(6)}=\left[R_{T(R)}^{2}+\right.$ $\left.\left(\xi_{T(R)}^{\left(n_{l^{\prime}, 5}\right)}\right)^{2}-2 R_{T(R)} \xi_{T(R)}^{\left(n_{l^{\prime}, 5}\right)} \cos \left(\phi_{T(R)}^{\left(n_{l^{\prime}, 5}\right)}-\phi_{T(R)}^{\left(n_{1, g_{1}\left(g_{2}\right)}\right)}\right)\right]^{1 / 2}$. Since the derivations of these angels are similar, only the derivation of $\phi_{S}^{\left(n_{1,1} n_{1,3}\right)}$ is given in Appendix B.

Based on the proposed MIMO V2V model, the channel characteristics in both spatial and temporal domains can be investigated. However, due to the page limit and considering the unique temporal characteristics of $\mathrm{V} 2 \mathrm{~V}$ channels, this paper focuses on the study of the complicated time variant properties, i.e., the Doppler PSD, which significantly affect the subcarrier orthogonality of OFDM systems. Under the wide-sense stationary uncorrelated-scattering condition and based on the proposed model in (1) and (3) with setting $\delta_{T}=\delta_{R}=0$, the Doppler PSD of channel impulse response $h_{p q}\left(t, \tau^{\prime}\right)$ of a V2V channel are completely determined by the Doppler PSD of $h_{l, p q}(t)$ in each tap since different taps are independent to each other. To simplify expressions, the subscript $\{\cdot\}_{p q}$ is omitted henceforth. Therefore, we can restrict our investigations to the following Doppler PSD as

$$
S_{h_{l} h_{l}}\left(f_{D}\right)=\int_{-\infty}^{\infty} \mathbf{E}\left[h_{l}(t) h_{l}^{*}(t-\tau)\right] e^{-j 2 \pi f_{D} \tau} d \tau
$$

where $f_{D}$ is the Doppler frequency, $(\cdot)^{*}$ denotes the complex conjugate operation, and $\mathbf{E}[\cdot]$ designates the statistical expectation operator. Since the LoS, single-, and doublebounced components are independent of each other, based on (1) we have the following Doppler PSD for the first $\operatorname{tap} S_{h_{1} h_{1}}\left(f_{D}\right)=S_{h_{1}^{L o S} h_{1}^{\operatorname{LoS}}}\left(f_{D}\right)+\sum_{i_{1}=1}^{I_{1}} S_{h_{1}^{S B_{i_{1}}} h_{1}^{S B_{i_{1}}}}\left(f_{D}\right)+$ $\sum_{i_{2}=1}^{I_{2}} S_{h_{1}^{D B_{i_{2}}} h_{1}^{D B_{i_{2}}}}\left(f_{D}\right)$. Whereas for other taps, according to (3) we have the Doppler PSD as $S_{h_{l^{\prime}} h_{l^{\prime}}}\left(f_{D}\right)=S_{h_{l^{\prime}}^{S B_{5}} h_{l^{\prime}}^{S B_{5}}}\left(f_{D}\right)+$ $\sum_{i_{2}=1}^{I_{2}} S_{h_{l^{\prime}}^{D B_{i_{2}}} h_{l^{\prime}}^{D B_{i_{2}}}}\left(f_{D}\right)$.

To derive the Doppler PSD, we assume the effective scatterers to be infinite, i.e., $N_{1, i_{1}}, N_{l^{\prime}, 5} \rightarrow \infty$, and apply the widely used von Mises distribution to characterize AoAs and AoDs [32]. In this case, the discrete angles $\phi_{T}^{\left(n_{1, i_{1}}\right)}$, $\phi_{T}^{\left(n_{l^{\prime}, 5}\right)}, \phi_{R}^{\left(n_{1, i_{1}}\right)}, \phi_{R}^{\left(n_{l^{\prime}, 5}\right)}, \phi_{S}^{\left(n_{1, \sigma_{i_{2}}} n_{1, \varsigma_{i_{2}}}\right)}$, and $\phi_{S}^{\left(n_{1, i_{2}} n_{l^{\prime}, 5}\right)}$ can be replaced by continuous random variables $\phi_{T}^{\left(1, i_{1}\right)}, \phi_{T}^{\left(l^{\prime}, 5\right)}$, $\phi_{R}^{\left(1, i_{1}\right)}, \phi_{R}^{\left(l^{\prime}, 5\right)}, \phi_{S}^{\left(\sigma_{i_{2}}, \varsigma_{i_{2}}\right)}$, and $\phi_{S}^{\left(i_{2}, 5\right)}$, respectively. The von Mises PDF is defined as $f(\phi) \triangleq \exp [k \cos (\phi-\mu)] /\left[2 \pi I_{0}(k)\right]$, where $\phi \in[-\pi, \pi), I_{0}(\cdot)$ is the zeroth-order modified Bessel function of the first kind, $\mu \in[-\pi, \pi)$ accounts for the mean value of the angle $\phi$, and $k(k \geq 0)$ is a real-valued parameter that controls the angle spread of the angle $\phi$. In this paper, for the angle of interest, i.e., the AoDs $\phi_{T}^{\left(n_{1,1(2)}\right)} \in[-\pi, \pi)$ and AoAs $\phi_{R}^{\left(n_{1,3(4)}\right)} \in[-\pi, \pi)$ for the two-ring model, and the AoAs $\phi_{R}^{\left(n_{l, 5}\right)} \in[-\pi, \pi)$ for the multiple confocal ellipse model, we use appropriate parameters ( $\mu$ and $k$ ) of the von Mises PDF as $\mu_{T}^{(1,1(2))}$ and $k_{T}^{(1,1(2))}, \mu_{R}^{(1,3(4))}$ and $k_{R}^{(1,3(4))}$, and $\mu_{R}^{(l, 5)}$ and $k_{R}^{(l, 5)}$, respectively. Applying the corresponding von Mises distribution, trigonometric transformations, and the equality $\int_{-\pi}^{\pi} \exp (a \sin c+b \cos c) d c=2 \pi I_{0}\left(\sqrt{a^{2}+b^{2}}\right)$ and $\int_{0}^{\infty} I_{0}\left(j \alpha \sqrt{x^{2}+y^{2}}\right) \cos (\beta x) d x=\cos \left(y \sqrt{\alpha^{2}-\beta^{2}}\right) / \sqrt{\alpha^{2}-\beta^{2}}$ [33], we can obtain the Doppler PSD of the LoS, single-, and double-bounced components as follows.

1) In the case of the LoS component

$S_{h_{1}^{L o S}} h_{1}^{\operatorname{LoS}}\left(f_{D}\right)=\frac{K}{K+1} \delta\left(f_{D}-\frac{v_{T}}{c} f_{c} \cos \gamma_{T}+\frac{v_{R}}{c} f_{c} \cos \gamma_{R}\right)$

where $\delta(\cdot)$ denotes the Dirac delta function.

2) In terms of the single-bounce two-ring model

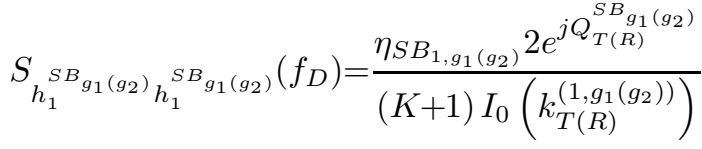

$$
\begin{aligned}
& \times \frac{\cos \left[F_{T(R)}^{S B_{g_{1}\left(g_{2}\right)}} \sqrt{\left(P \bar{v}_{g_{1}\left(g_{2}\right)}^{T(R)}\right)^{2}-\left(E_{T(R)}^{\left.S B_{g_{1}\left(g_{2}\right)}\right)^{2}}\right]}\right.}{\sqrt{\left(P * \bar{v}_{g_{1}\left(g_{2}\right)}^{T(R)}\right)^{2}-\left(E_{T(R)}^{\left.S B_{g_{1}\left(g_{2}\right)}\right)^{2}}\right.}}
\end{aligned}
$$

where $P=2 \pi f_{c} / c, Q_{T(R)}^{S B_{g_{1}\left(g_{2}\right)}}=\frac{k_{T(R)}^{\left(1, g_{1}\left(g_{2}\right)\right)} \cos \mu_{T(R)}^{\left(1, g_{1}\left(g_{2}\right)\right)}}{j \bar{v}_{g_{1}\left(g_{2}\right)}^{T(R)}}$, $E_{T(R)}^{S B_{g_{1}\left(g_{2}\right)}}=\frac{k_{T(R)}^{\left(1, g_{1}\left(g_{2}\right)\right)} \sin \mu_{T(R)}^{\left(1, g_{1}\left(g_{2}\right)\right)}}{j P \bar{v}_{g_{1}\left(g_{2}\right)}^{T(R)}}$, and $E_{T(R)}^{S B_{g_{1}\left(g_{2}\right)}}=2 \pi f_{D}+$ $P \bar{v}_{g_{1}\left(g_{2}\right)}^{R(T)}$.

3) In the case of the single-bounce multiple confocal ellipses 
model

$$
\begin{aligned}
& S_{h_{l}^{S B_{5}} h_{l}^{S B_{5}}}(\tau)=\frac{\eta_{S B_{l, 5}}}{2 \pi I_{0}\left(k_{R}^{(l, 5)}\right) U} \int_{-\infty}^{\infty} \int_{-\pi}^{\pi} e^{k_{R}^{(l, 5)} \cos \left(\phi_{R}^{(l, 5)}-\mu_{R}^{(l, 5)}\right)} \\
& \times e^{j \frac{2 \pi f_{c} \tau}{c}\left[\bar{v}_{5}^{T} \cos \phi_{T}^{(l, 5)}+\bar{v}_{5}^{R} \cos \phi_{R}^{(l, 5)}\right]} e^{-j 2 \pi f_{D} \tau} d \phi_{R}^{(l, 5)} d \tau
\end{aligned}
$$

where $U=K+1$ only appears for the first tap.

4) In terms of the double-bounce component for the first tap, we have the expression of $S_{h_{1}^{D B_{i_{2}}} h_{1}^{D B_{i_{2}}}}(\tau)$ as (9) on the next page.

5) In terms of the double-bounce component for other taps, the expression of $S_{h_{l^{\prime}}{ }^{D B_{g_{1}\left(g_{2}\right)}} h_{l^{\prime}}{ }^{D B_{g_{1}\left(g_{2}\right)}}}(\tau)$ can be shown as (10) on the next page.

For the Doppler PSD in (5), the range of Doppler frequency is limited by $\left|f_{D}\right| \leq\left[v_{T}+2 \max \left\{v_{S T_{1}}, v_{S T_{2}}\right\}+\right.$ $\left.2 \max \left\{v_{S R_{1}}, v_{S R_{2}}\right\}+v_{R}\right] f_{c} / c$. Finally, the Doppler PSD of the channel impulse response $h\left(t, \tau^{\prime}\right)$ can be expressed as $S_{h h}\left(f_{D}\right)=\sum_{l=1}^{L} c_{l}^{2} S_{h_{l} h_{l}}\left(f_{D}\right)$.

\section{Precoding Based CAncellation Scheme}

\section{A. Two Currently Important ICI Cancellation Methods}

In order to mitigate the impact of ICI, several ICI cancellation methods have been proposed. Among them two types of ICI cancellation methods, i.e., ICI self cancellation and two-path transmission, have been widely accepted due to their simplicity and effectiveness in ICI mitigation.

1) ICI Self-Cancellation Method: ICI self-cancellation method can efficiently combat ICI by employing data repetition within one OFDM symbol interval. As depicted in Fig. 3 (a), the additional processes for the ICI self-cancellation method with respect to the normal OFDM procedure are the ICI self-cancellation module, including the ICI canceling modulation before the inverse fast Fourier transform (IFFT) operation at the $\mathrm{Tx}$ and the ICI canceling demodulation after the fast Fourier transform (FFT) operation at the Rx [16].

For the ICI canceling modulation, the input modulated symbols $\{X\}$ are first grouped into several transmit blocks each consisting of $\frac{N}{2}$ modulated symbols, which are then mapped onto the $N$ subcarriers using one-to-two mapping rule:

$$
\tilde{X}_{\mathcal{M}\{k\}}=\left\{\begin{array}{c}
X_{k}, \quad k=0, \ldots, \frac{N}{2}-1 \\
\mathcal{O}\left(X_{k-\frac{N}{2}}\right), \quad k=\frac{N}{2}, \ldots, N-1
\end{array}\right.
$$

where $\left\{\widetilde{X}_{k}\right\}_{k=0}^{N-1}$ are the real transmitted symbols on the OFDM subcarriers, $\mathcal{M}$ is a mapping set whose elements are chosen according to the specific mapping criterion, such as adjacent mapping, symmetric mapping, and mirror mapping, and $\mathcal{O}(x)$ is defined as the mapping operation which reflects the relationship between the two symbols with the same information to be mapped on the subcarrier pair. In ICI selfcancellation methods, conversion and conjugate relationships are commonly utilized and can be represented in (11) as $\mathcal{O}(x)=-x$ and $\mathcal{O}(x)=x^{*}$, respectively. Therefore, there are six ICI self-cancellation schemes with the combination of the three mapping criteria and two mapping operations: adjacent symbol repetition (ASR) [17], adjacent conjugate symbol repetition (ACSR) [19], symmetric symbol repetition (SSR) [20], symmetric conjugate symbol repetition (SCSR) [21], mirror symbol repetition (MSR), and mirror conjugate symbol repetition (MCSR) [22]. Taking the mirror mapping criterion with the conjugate mapping operation, i.e., MCSR scheme, as an example, we have $\mathcal{M}=\left\{1, \ldots, \frac{N}{2}-1, \emptyset, N-1, \ldots, \frac{N}{2}+\right.$ $1, \emptyset\}$ with $\emptyset$ denoting invalid mapping. Therefore, the final transmitted symbols in the frequency domain after the ICI self-cancellation module will be $\widetilde{X}_{1}=\widetilde{X}_{N-1}^{*}=X_{0}, \widetilde{X}_{2}=$ $\widetilde{X}_{N-2}^{*}=X_{1}, \ldots, \widetilde{X}_{N / 2-1}=\widetilde{X}_{N / 2+1}^{*}=X_{N / 2-2}, \widetilde{X}_{0}=$ $\widetilde{X}_{N / 2}=0$ [22].

After the ICI canceling modulation, the received signals on subcarrier $m$ and its corresponding mapped subcarrier pair $m^{\prime}$ ( $m^{\prime}=m+1$ for the adjacent mapping criterion, $m^{\prime}=$ $N-m-1$ for the symmetric mapping criterion, or $m^{\prime}=$ $N-m$ for the mirror mapping criterion) will carry on the same data symbol. This signal redundancy makes it possible to further improve the ICI mitigation performance through a combination technique, which can be realized as

$$
\begin{aligned}
& \widehat{X}_{m}=\frac{\mathcal{O}\left(H_{\mathcal{M}\left\{m+\frac{N}{2}\right\}, \mathcal{M}\left\{m+\frac{N}{2}\right\}}^{*} Y_{\mathcal{M}\left\{m+\frac{N}{2}\right\}}\right)}{\left|H_{\mathcal{M}\{m\}, \mathcal{M}\{m\}}\right|^{2}+\left|H_{\mathcal{M}\left\{m+\frac{N}{2}\right\}, \mathcal{M}\left\{m+\frac{N}{2}\right\}}\right|^{2}}+ \\
& \frac{H_{\mathcal{M}\{m\}, \mathcal{M}\{m\}}^{*} Y_{\mathcal{M}\{m\}}}{\left|H_{\mathcal{M}\{m\}, \mathcal{M}\{m\}}\right|^{2}+\left|H_{\mathcal{M}\left\{m+\frac{N}{2}\right\}, \mathcal{M}\left\{m+\frac{N}{2}\right\}}\right|^{2}}
\end{aligned}
$$

where $H_{m, k}=\frac{1}{N} \sum_{l=1}^{L} F_{l}(m-k) e^{-j \frac{2 \pi l k}{N}}, m=0, \ldots, \frac{N}{2}-1$, $F_{l}(z)=\sum_{n=0}^{N-1} h_{l}^{(n)} e^{-j \frac{2 \pi n z}{N}}$, and $h_{l}^{(n)}$ is the $l$-th sample of the time-varying channel impulse response at time instant $\frac{n T_{s}}{N}$ with $T_{s}$ denoting the symbol duration.

2) Two-Path Transmission Method: Unlike the ICI selfcancellation method where the data is repeated within one OFDM block, the two-path transmission method transmits the data copies in two concatenated OFDM blocks, which are usually referred to as two independent paths separated by time division multiplexing (TDM), as shown in Fig. 3 (b). Similar to the ICI self-cancellation method, by applying the combination technique on the received signals from both paths at the Rx, the ICI generated from one path can be significantly mitigated by that generated from the other path.

Currently, PC and CC schemes have been known as the typical two-path cancellation schemes. Taking the CC scheme as an example, the first path follows the normal OFDM procedure, while the second path adopts a conjugate of the normal OFDM signal both before the transmission and after the reception, as illustrated in Fig. 3(b), without considering the $e^{j \phi}$ and $-e^{j \phi}$ modules. Finally, the received signals at the $m$-th subcarrier for both paths are combined as (13) by setting $\phi=0$, where the effect of noise is ignored. More recently, to further improve the ICI mitigation performance of CC scheme, the PRCC scheme has been proposed by using PRA method that adds an artificial phase rotation of $\phi$ for both paths at the Tx in the CC scheme, as shown in Fig. 3(b). However, the performance of PRCC scheme is highly dependent on the accuracy of the chosen phase rotation, which is related to the 


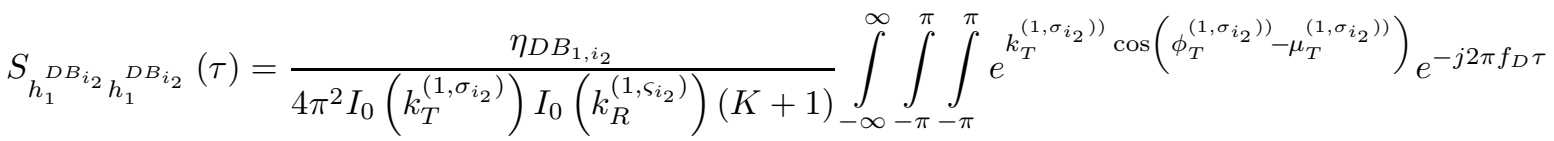

$$
\begin{aligned}
& e^{k_{R}^{\left.\left(1, \varsigma_{i_{2}}\right)\right)} \cos \left(\phi_{R}^{\left.\left(1, \varsigma_{i_{2}}\right)\right)}-\mu_{R}^{\left.\left(1, \varsigma_{i_{2}}\right)\right)}\right) e^{j \frac{2 \pi f_{c} \tau}{c}}\left[\bar{v}_{\sigma_{i_{2}}}^{T} \cos \phi_{T}^{\left(n_{1, \sigma_{i_{2}}}\right)}+\bar{v}_{\sigma_{i_{2}}, \varsigma_{i_{2}}}^{S} \cos \phi_{S}^{\left(n_{1, \sigma_{i_{2}}} n_{1, \varsigma_{i_{2}}}\right)}+\bar{v}_{\varsigma_{i_{2}}}^{R} \cos \phi_{R}^{\left(n_{1, \varsigma_{i_{2}}}\right)}\right]} d \phi_{T}^{\left(1, \sigma_{i_{2}}\right)} d \phi_{R}^{\left(1, \varsigma_{i_{2}}\right)} d \tau
\end{aligned}
$$

$$
\begin{aligned}
& S_{h_{l^{\prime}}^{D B_{g_{1}\left(g_{2}\right)}} h_{l^{\prime}}^{D B} g_{g_{1}\left(g_{2}\right)}}(\tau)=\frac{\eta_{D B_{l^{\prime}, g_{1}\left(g_{2}\right)}}}{4 \pi^{2} I_{0}\left(k_{T(R)}^{\left(1\left(l^{\prime}\right), g_{1}(5)\right)}\right) I_{0}\left(k_{R}^{\left(1, g_{2}\right)}\right)(K+1)}
\end{aligned}
$$

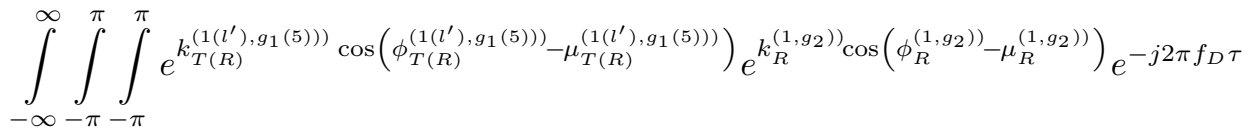

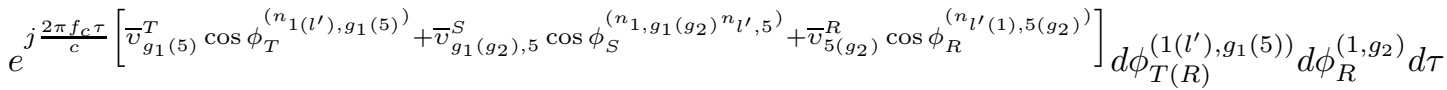

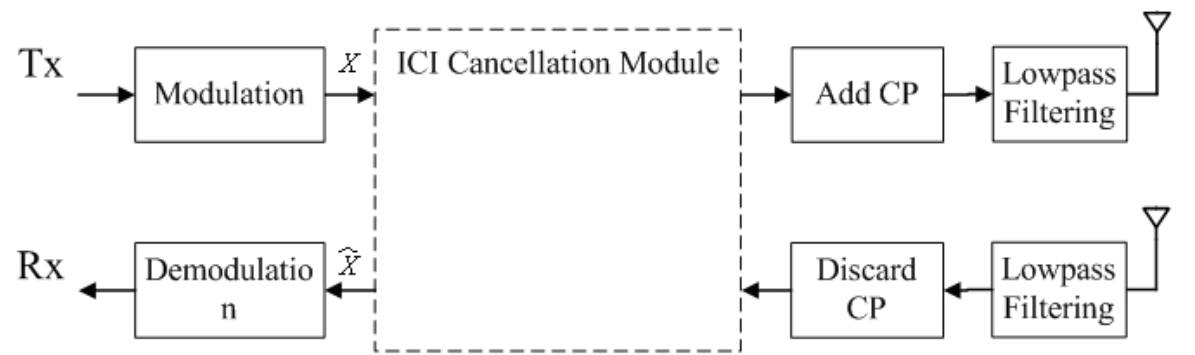

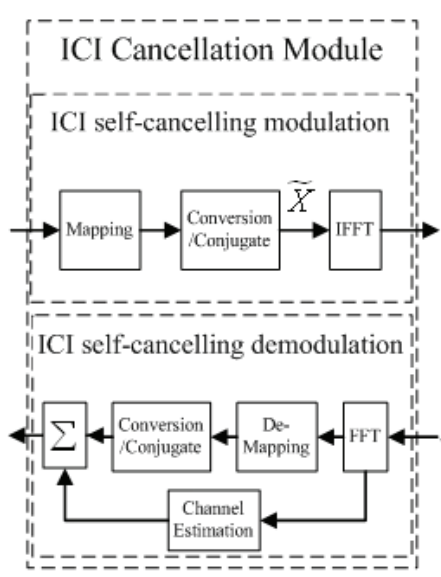

(a)

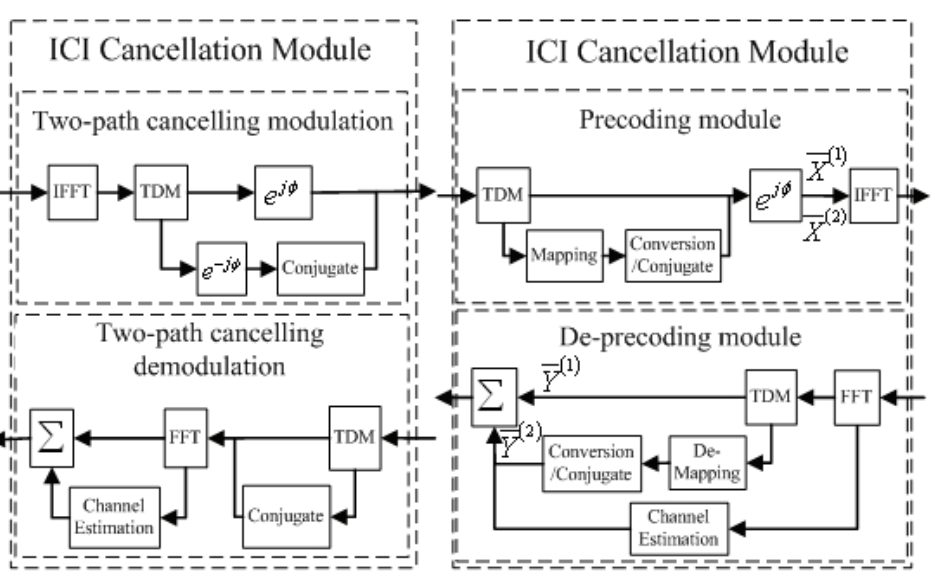

(b)

(c)

Fig. 3. Block diagram of a OFDM transceiver with (a) ICI self-cancellation module, (b) PRCC two-path cancellation module, and (c) PBC with PRA method module.

real frequency offset experienced at the Rx and thus needs a precise estimation at the Rx and an error-free feedback link to the Tx. For example, the phase rotation adopted at the Tx is derived as $\phi=-\pi \hat{\varepsilon}\left(1-\frac{1}{N}\right)$, where $\hat{\varepsilon}$ is the feedback estimated frequency offset from the Rx in [25]. The PRA method used in the PRCC scheme aims to make the phases of the ICI coefficients for the both paths have an opposite polarity, so that they can cancel out each other when the combination of the both paths is implemented. Therefore, the combination of received signals at the $m$-th subcarrier for both paths can be expressed as

$$
\begin{aligned}
& \widehat{X}_{m}=\frac{e^{j \phi}\left|H_{m, m}\right|^{2}+e^{-j \phi}\left|H_{-m,-m}\right|^{2}}{\left|H_{m, m}\right|^{2}+\left|H_{-m,-m}\right|^{2}} X_{m}+ \\
& \frac{\sum_{k=0, k \neq m}^{N-1}\left(e^{j \phi} H_{m, m}^{*} H_{m, k}+e^{-j \phi} H_{-m,-m} H_{-m,-k}^{*}\right) X_{k}}{\left|H_{m, m}\right|^{2}+\left|H_{-m,-m}\right|^{2}} .
\end{aligned}
$$

It has been shown in [25] that due to the application of the PRA method, the PRCC scheme outperforms most existing ICI cancellation schemes under an ideal assumption 
that the accurate phase rotation is obtained at the Tx. Note that the PRA method proposed in the PRCC scheme can be also applicable to any ICI self-cancellation and two-path cancellation schemes for further improving their ICI mitigation performance, although it has not been specified in [25].

\section{B. Precoding Based Cancellation Method}

From the previous subsection, it is clear that the essence of the ICI self-cancellation and two-path transmission schemes is the proper design of the redundant signals associated with the combination technique. Specifically, the redundant signals for ICI self-cancellation schemes are introduced within one OFDM block, while those for two-path transmission schemes are in two concatenated OFDM blocks. Compared with the normal OFDM procedure, the complexity increase of ICI selfcancellation schemes is marginal. However, when the ICI selfcancellation schemes are applied into the IEEE $802.11 \mathrm{p}$ based V2V systems, performance degradation will appear as the configuration of the OFDM block, which includes not only data symbols but also pilot symbols, seriously breaks the mapping criterion to the data symbols. Fortunately, this problem can be successfully avoided by the application of the two-path transmission schemes due to no additional operation within one OFDM block. The two separate OFDM transceivers, however, will significantly increase the realization complexity of two-path transmission schemes and as a consequence limit the practical use of the two-path transmission schemes. This motivates us to propose a new type of ICI cancellation method that owns both the advantages of the ICI self-cancellation method and two-path cancellation method and meanwhile is suitable for real V2V systems.

In [26], we have discovered a relationship between ICI selfcancellation schemes and two-path transmission schemes and proved that the $\mathrm{CC}$ scheme is actually another implementation manner of the MCSR scheme. Motivated by this, a new type of ICI cancellation method is proposed where the main additional operations compared with normal OFDM systems have been integrated inside the precoding and de-precoding modules, as shown in Fig. 3(c), without considering the $e^{j \phi}$ module and thus named as PBC scheme. The PBC scheme involves the redundant signals by properly applying the mapping criterion and mapping operation in two concatenated OFDM blocks separated by TDM. For the precoding module, one OFDM block input $\left\{X_{k}\right\}_{k=0}^{N-1}$ will become two OFDM blocks output $\left\{\bar{X}_{k}^{(1)}\right\}_{k=0}^{N-1}$ and $\left\{\bar{X}_{k}^{(2)}\right\}_{k=0}^{N-1}$, where the first OFDM block $\left\{\bar{X}_{k}^{(1)}\right\}_{k=0}^{N-1}$ is equal to the input OFDM block, i.e., $\bar{X}_{k}^{(1)}=$ $X_{k}$ and the second OFDM block $\left\{\bar{X}_{k}^{(2)}\right\}_{k=0}^{N-1}$ is obtained by following the mapping rule $\bar{X}_{\mathcal{M}\{k\}}^{(2)}=\mathcal{O}\left(X_{k}\right)$. Similar to (11), $\mathcal{M}$ is a mapping set whose elements are chosen according to the specific mapping criterion, such as adjacent mapping with $\mathcal{M}=\{1,2, \ldots, N-1,0\}$, symmetric mapping with $\mathcal{M}=\{N-1, N-2, \ldots, 0\}$, and mirror mapping with $\mathcal{M}=\{0, N-1, N-2, \ldots, 1\}$, and $\mathcal{O}(x)$ is defined as the mapping operation which is either conversion operation or conjugate operation. Therefore, similar to ICI self-cancellation schemes, in total there are six PBC schemes named as: precoding based ASR (PB-ASR), PB-ACSR, PB-SSR, PBSCSR, PB-MSR, and PB-MCSR. Finally, the combination of the received signals at the $m$-th subcarrier for both OFDM blocks can be derived as

$$
\widehat{X}_{m}=\frac{H_{m, m}^{*} \bar{Y}_{m}^{(1)}+\mathcal{O}\left\{H_{\mathcal{M}\{m\}, \mathcal{M}\{m\}}^{*} \bar{Y}_{\mathcal{M}\{m\}}^{(2)}\right\}}{\left|H_{m, m}\right|^{2}+\left|H_{\mathcal{M}\{m\}, \mathcal{M}\{m\}}\right|^{2}} .
$$

where $\left\{\bar{Y}_{m}^{(1)}\right\}_{m=0}^{N-1}$ and $\left\{\bar{Y}_{m}^{(2)}\right\}_{m=0}^{N-1}$, respectively, are the received signals corresponding to the transmitted two OFDM blocks in the frequency domain.

In Appendix C, we prove that the ICI self-cancellation schemes express the same ICI mitigation performance as the PBC schemes. However, by properly applying the mapping criterion and mapping operation into two concatenated OFDM blocks rather than into one OFDM block as in ICI selfcancellation schemes, the proposed PBC schemes can be directly implemented into real V2V systems without exhibiting any performance degradation. Moreover, unlike two-path concellation schemes that design the two concatenated OFDM blocks after the IFFT at the Tx and before the FFT at the Rx, as illustrated in Fig. 3(b), the design of the two OFDM blocks in the $\mathrm{PBC}$ scheme is integrated in the precoding module before the IFFT at the Tx and the de-precoding module after the FFT at the Rx, as shown in Fig. 3(c). This indicates that the precoding and de-precoding operations can be accomplished via a little software programming effort without modifying the hardware of present OFDM systems as previous two-path cancellation schemes have to do. Therefore, by properly taking the both advantages of ICI self-cancellation schemes and twopath cancellation schemes, the proposed PBC schemes can be easily implemented into real V2V systems and have no any performance degradation.

Similarly, the PRA method can be also used in the PBC scheme for further improving its ICI mitigation performance as shown in Fig. 3(c). Based on the proof in Appendix C and conclusions in [22] [26], it allows us to conclude that the PB-MCSR with the PRA method, named as phase rotated PB-MCSR (PRPB-MCSR), has the same performance as the PRCC scheme. Therefore, the PRPB-MCSR is the best ICI cancellation scheme for real V2V systems under the ideal assumption that the accurate phase rotation is available at the Tx.

\section{Constant Phase Rotation Method}

The key issue on the implementation of the PRA method is how to obtain the accurate artificial phase rotation at the Tx. In general, the implementation of the PRA method needs a powerful frequency offset estimator at the $\mathrm{Rx}$ and an error-free feedback link from the $\mathrm{Rx}$ to the $\mathrm{Tx}$, which will significantly increase the system complexity and reduce the system real-time performance. Due to the complicated moving environments of real $\mathrm{V} 2 \mathrm{~V}$ systems, the frequency offset caused by Doppler should be a spectrum largely spread in a wide range rather than a single value. Therefore, a precise estimation of the frequency offset in real V2V systems will not only be a serious burden at the Rx but also hardly be obtained. Moreover, the PRA method is derived based on a single-valued frequency offset and thus may not be suitable for real V2V systems. 
The above gap motivates us to propose a CPRA method where the artificial phase rotation is constant and irrelevant to the frequency offset. Therefore, the implementation of the proposed CPRA method is simple and only needs a $e^{j \phi}$ module with a given constant optimal phase rotation $\phi$ at the Tx. More importantly, the constant phase rotation is designed over a given frequency offsets range rather than based on a single-valued frequency offset. The CIR is used to design the CPRA method. Therefore, the basic design rule is that the CIR of any ICI cancellation scheme with the designed CPRA method should overwhelm the CIR of the normal OFDM systems and meanwhile approach the CIR of the ICI cancellation scheme with the PRA method for any given fixed frequency offset. By taking the PRPB-MCSR as an example, this section details the design procedure of the CPRA method. Considering a single-valued frequency offset $\Delta f=f_{d}$ where $f_{d}$ denotes any Doppler shift in the Doppler spectrum of $h_{l}^{(n)}$, the CIRs of normal OFDM systems and constant phase rotation PB-MCSR (CPRPB-MCSR) with a phase rotation $\phi$ can be expressed as [26]

$$
C_{O F D M}=\frac{\operatorname{sinc}^{2}(\varepsilon)}{1-\operatorname{sinc}^{2}(\varepsilon)}
$$

and

$$
C_{C P R P B-M C S R}=\frac{\operatorname{sinc}^{2}(\varepsilon) \cos ^{2}\left(\pi \varepsilon\left(1-\frac{1}{N}\right)+\phi\right)}{\frac{1}{2}+\frac{1}{2} \operatorname{sinc}(2 \varepsilon) X-\operatorname{sinc}^{2}(\varepsilon) Y}
$$

respectively, where $X=\cos \left(2 \pi \varepsilon\left(1-\frac{1}{N}\right)+2 \phi\right), Y=$ $\cos ^{2}\left(\pi \varepsilon\left(1-\frac{1}{N}\right)+\phi\right), \operatorname{sinc}(x)=\sin (\pi x) / \pi x$, and the normalized frequency offset $\varepsilon=\Delta f T_{s}$. The CIR of the PRPB-MCSR can be obtained directly from (16) by setting $\phi=-\pi \varepsilon\left(1-\frac{1}{N}\right)$ as

$$
C_{P R P B-M C S R}=\frac{\operatorname{sinc}^{2}(\varepsilon)}{\frac{1}{2}+\frac{1}{2} \operatorname{sinc}(2 \varepsilon)-\operatorname{sinc}^{2}(\varepsilon)}
$$

which is actually the maximum of (16). Note that for the PRPB-MCSR, the artificial phase rotation is relevant to the normalized frequency offset $\varepsilon$.

\section{A. The Range of Feasible Solutions of $\phi$}

Dividing the nominator and denominator in (16) by $\cos ^{2}\left(\pi \varepsilon\left(1-\frac{1}{N}\right)+\phi\right)$, it is observed that the only difference among (15)-(17) lies in the denominator. Therefore, to guarantee that the CIR in (16) is larger than that in (15) for a given frequency offset range $\varepsilon \in\left[\varepsilon_{\min }, \varepsilon_{\max }\right]$, where $\varepsilon_{\min }>0$ and $\varepsilon_{\max } \leq 0.5$ are the minimum and maximum normalized frequency offsets, respectively, we have

$$
\begin{aligned}
\psi(\varepsilon, \phi)<1, \psi(\varepsilon, \phi) & =\frac{1+\operatorname{sinc}(2 \varepsilon) \cos \left(2 \pi \varepsilon\left(1-\frac{1}{N}\right)+2 \phi\right)}{2 \cos ^{2}\left(\pi \varepsilon\left(1-\frac{1}{N}\right)+\phi\right)} \\
& \approx \frac{1+\operatorname{sinc}(2 \varepsilon) \cos (2 \pi \varepsilon+2 \phi)}{1+\cos (2 \pi \varepsilon+2 \phi)}
\end{aligned}
$$

where $\varepsilon \in\left[\varepsilon_{\min }, \varepsilon_{\max }\right]$ and the approximation is derived based on the reasonable assumption of a large value of $N$. Calculating the above inequality, we have $\cos (2 \pi \varepsilon+2 \phi)(1-$ $\operatorname{sinc}(2 \varepsilon))>0$. Resorting to the property $\operatorname{sinc}(2 \varepsilon)<1$, we can obtain feasible solutions of $\phi$ over the range as $\left[-\frac{\pi}{4}-\pi \varepsilon_{\min }+k \pi, \frac{\pi}{4}-\pi \varepsilon_{\max }+k \pi\right]$ with $k$ being an integer.
Without loss of generality, in the sequel, $\phi$ is constrained in its principal value interval $\phi \in\left[-\frac{\pi}{4}-\pi \varepsilon_{\min }, \frac{\pi}{4}-\pi \varepsilon_{\max }\right]$.

\section{B. Derivation of Optimal $\phi$}

Based on the basic design rule, the optimal $\phi$ should be chosen such that the CIR for any $\varepsilon$ drawn from the possible frequency offset range experienced at the Rx approaches the saturation level, i.e., the CIR of the PRPB-MCSR. Therefore, if we define a metric $\mathscr{D}$ which characterizes the difference between the CIR of the CPRPB-MCSR and the CIR of the PRPB-MCSR for a given $\varepsilon$, the optimal $\phi$ will be figured out by minimizing the accumulated differences of CIRs with $\varepsilon$ spreading from $\varepsilon_{\min }$ to $\varepsilon_{\max }$

$$
\begin{aligned}
& \phi_{\text {opt }}=\min \{\rho(\phi)\}, \rho(\phi)= \\
& \int_{\varepsilon_{\min }}^{\varepsilon_{\max }} \mathscr{D}\left(C_{C P R P B-M C S R}(\varepsilon, \phi), C_{P R P B-M C S R}(\varepsilon)\right) d \varepsilon .
\end{aligned}
$$

where $\mathscr{D}\left(C_{C P R P B-M C S R}(\varepsilon, \phi), C_{P R P B-M C S R}(\varepsilon)\right)=$ $\left|C_{P R P B-M C S R}(\varepsilon) \quad-C_{C P R P B-M C S R}(\varepsilon, \phi)\right|^{2}$ is the square error between these two CIRs and $\phi \in\left[-\frac{\pi}{4}-\pi \varepsilon_{\min }, \frac{\pi}{4}-\pi \varepsilon_{\max }\right]$. The integration range $\left[\varepsilon_{\min }, \varepsilon_{\max }\right]$ should be determined by the transceiver performance and communication environments as the former determines the level of phase noise and frequency mismatch between the received signal and local oscillator, and the latter determines the level of Doppler spectrum. The optimal $\phi_{\text {opt }}$ can be readily obtained by minimizing $\rho(\phi)$ through computer numerical calculating. Especially, if $\left[\varepsilon_{\min }, \varepsilon_{\max }\right]=[0,0.5]$, the feasible solutions of $\phi$ are reduced to one element, $\phi=-\frac{\pi}{4}$, which actually turns out to be the optimal phase rotation.

So far, it is clear that the phase rotation of the PRA method is the optimal one for any given single-valued $\varepsilon$, while the phase rotation of the CPRA method is the suboptimal one for any given single-valued $\varepsilon$ but is the optimal one over a given frequency offset range $\left[\varepsilon_{\min }, \varepsilon_{\max }\right]$. Since the Doppler spectrum for real V2V systems in practical results in a wider spread frequency offset range rather than a singlevalued frequency offset, compared with the PRA method, the CPRA method should express better performance and more importantly has very small implementation complexity.

\section{Simulation Results And Analysis}

Unless otherwise specified, all the channel related parameters used in this section are listed in Table II. A real V2V system following the IEEE $802.11 \mathrm{p}$ standard was used with basic parameters as follows [13]. OFDM subcarrier number is $N=64$, CP length is $L p=16$, both short and long training preambles consisting of 2 OFDM symbols were transmitted before data frame for the signal synchronization, frequency offset estimation, and channel estimation, 4 pilot symbols are multiplexed with the transmitted data in the frequency domain for phase tracking. Least squares (LS) estimator is used for the channel estimation.

Figs. 4 and 5 show the Doppler PSDs of the proposed V2V model for the first and second taps with different VTDs (low and high) when the Tx and Rx move in opposite directions 
TABLE II

CHANNEL RELATED PARAMETERS USED IN SIMULATIONS

\begin{tabular}{|c|c|c|c|}
\hline All scenarios & & asic parameters & $\begin{array}{l}f_{c}=5.9 \mathrm{GHz}, v_{T}=v_{R}=v_{S T_{1(2)}}=v_{S R_{1(2)}}=105 \mathrm{~km} / \mathrm{h}, D=300 \mathrm{~m}, \\
\mathrm{~L}=4,\left[a_{1}, a_{2}, a_{3}, a_{4}\right]=[160,175,190,205] \mathrm{m}, R_{T}=R_{R}=7 \mathrm{~m}, \\
{\left[c_{1}, c_{2}, c_{3}, c_{4}\right]=[\sqrt{0.88}, \sqrt{0.08}, \sqrt{0.03}, \sqrt{0.01}],\left\{N_{1, i_{1}}\right\}_{i_{1}=1}^{5}=N_{2,5}=N_{3,5}=N_{4,5}=30}\end{array}$ \\
\hline \multirow{6}{*}{$\begin{array}{l}\text { Tx and } \mathrm{Rx} \\
\text { move in opposite } \\
\text { directions (OD) }\end{array}$} & \multirow{3}{*}{$\begin{array}{l}\text { LVTD } \\
\text { scenario }\end{array}$} & Rician factor & $K=3.786$ \\
\hline & & $\begin{array}{l}\text { environment-related } \\
\text { parameters }\end{array}$ & $\begin{array}{l}\text { Two-ring: } k_{T}^{(1,1}=26.4, k_{T}^{(1,2}=25.6, k_{R}^{(1,3}=27.2, k_{R}^{(1,4} \underline{\underline{L}}=28.9 \\
\mu_{T}^{(1,1)}=7^{\circ}, \mu_{T}^{(1,2)}=133^{\circ}, \mu_{R}^{(1,3)}=172^{\circ}, \mu_{R}^{(1,4}=52^{\circ} \\
\text { First ellipse: } k_{R}^{(1,5)}=6.5, \mu_{R}^{(1,5}=159^{\circ} ; \text { Second ellipse: } k_{R}^{(2,5)}=7.6, \mu_{R}^{(2,5)}=63^{\circ} \\
\text { Third ellipse: } k_{R}^{(3,5}=8.7, \mu_{R}^{(3,5}=-136^{\circ} ; \text { Fourth ellipse: } k_{R}^{(4,5}=9.4, \mu_{R}^{(4,5}=-43^{\circ}\end{array}$ \\
\hline & & $\begin{array}{l}\text { energy-related } \\
\text { parameters }\end{array}$ & $\begin{array}{l}\text { First tap: } \eta_{D B_{1,1}}=\eta_{D B_{1,2}}=\eta_{D B_{1,3}}=\eta_{D B_{1,4}}=0.03 \\
\eta_{S B_{1,1}}=\eta_{S B_{1,2}}=\eta_{S B_{1,3}}=\eta_{S B_{1,4}}=0.002, \eta_{S B_{1,5}}=0.872 \\
\text { Second tap: } \eta_{D B_{2,1}}=\eta_{D B_{2,2}}=\eta_{D B_{2,3}}=\eta_{D B_{2,4}}=0.02, \eta_{S B_{2,5}}=0.92 \\
\text { Third tap and fourth tap are the same as the second tap. }\end{array}$ \\
\hline & \multirow{3}{*}{$\begin{array}{l}\text { HVTD } \\
\text { scenario }\end{array}$} & Rician factor & $K=0.156$ \\
\hline & & $\begin{array}{l}\text { environment-related } \\
\text { parameters }\end{array}$ & $\begin{array}{l}\text { Two-ring: } k_{T}^{(1,1}=4.6, k_{T}^{(1,2}=5.8, k_{R}^{(1,3}=5.2, k_{R}^{(1,4}=4.3 \\
\mu_{T}^{(1,1)}=7^{\circ}, \mu_{T}^{(1,2)}=133^{\circ}, \mu_{R}^{(1,3}=172^{\circ}, \mu_{R}^{(1,4)}=52^{\circ} \\
\text { Other parameters are the same as those of the OD-LVTD scenario. }\end{array}$ \\
\hline & & $\begin{array}{l}\text { energy-related } \\
\text { parameters }\end{array}$ & 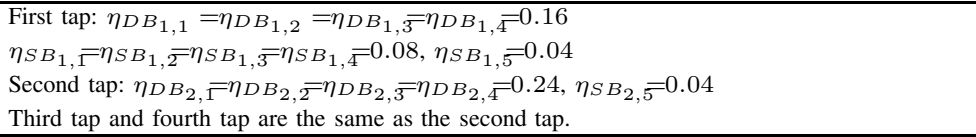 \\
\hline \multirow{6}{*}{$\begin{array}{l}\text { Tx and Rx } \\
\text { move in the } \\
\text { same direction }(\mathrm{SD})\end{array}$} & \multirow{3}{*}{$\begin{array}{c}\text { LVTD } \\
\text { scenario }\end{array}$} & Rician factor & $K=2.168$ \\
\hline & & $\begin{array}{l}\text { environment-related } \\
\text { parameters }\end{array}$ & $\begin{array}{l}\text { Two-ring: } k_{T}^{(1,1}=22.6, k_{T}^{(1,2}=23.4, k_{R}^{(1,3}=23.6, k_{R}^{(1,4}=22.9 \\
\mu_{T}^{(1,1)}=6^{\circ}, \mu_{T}^{(1,2)}=83^{\circ}, \mu_{R}^{(1,3)}=11^{\circ}, \mu_{R}^{(1,4)}=97^{\circ} \\
\text { First ellipse: } k_{R}^{(1,5)}=5.6, \mu_{R}^{(1,5}=69^{\circ} ; \text { Second ellipse: } k_{R}^{(2,5)}=6.3, \mu_{R}^{(2,5} \underline{L}=123^{\circ} \\
\text { Third ellipse: } k_{R}^{(3,5)}=7.4, \mu_{R}^{(3,5}=-166^{\circ} ; \text { Fourth ellipse: } k_{R}^{(4,5}=8.2, \mu_{R}^{(4,5}=-56^{\circ}\end{array}$ \\
\hline & & $\begin{array}{l}\text { energy-related } \\
\text { parameters }\end{array}$ & $\begin{array}{l}\text { First tap: } \eta_{D B_{1,1}}=\eta_{D B_{1,2}}=\eta_{D B_{1,3}}=\eta_{D B_{1,4}}=0.06 \\
\eta_{S B_{1,1}}=\eta_{S B_{1,2}}=\eta_{S B_{1,3}}=\eta_{S B_{1,4}}=0.001, \eta_{S B_{1,5}}=0.756 \\
\text { Second tap: } \eta_{D B_{2,1}}=\eta_{D B_{2,2}}=\eta_{D B_{2,3}}=\eta_{D B_{2,4}}=0.01, \eta_{S B_{2,5}}=0.96 \\
\text { Third tap and fourth tap are the same as the second tap. }\end{array}$ \\
\hline & \multirow{3}{*}{$\begin{array}{l}\text { HVTD } \\
\text { scenario }\end{array}$} & Rician factor & $K=0.156$ \\
\hline & & $\begin{array}{l}\text { environment-related } \\
\text { parameters }\end{array}$ & $\begin{array}{l}\text { Two-ring related parameters are the same as those of the OD-HVTD scenario. } \\
\text { Other parameters are the same as those of the SD-LVTD scenario. }\end{array}$ \\
\hline & & energy-related parameters & Parameters are the same as those of the OD-HVTD scenario \\
\hline
\end{tabular}

(OD) and same direction (SD), respectively. For comparison purposes, the measured Doppler PSDs taken from Figs. 4(a)(d) in [1] are also plotted in Figs. 4(a), (b) and Figs. 5(a), (b), respectively. In [1], the measurement campaigns were performed at a carrier frequency of $5.9 \mathrm{GHz}$ on an expressway with a LVTD. The distance between the Tx and Rx was approximately $D=300 \mathrm{~m}$ and the directions of movement were $\gamma_{T}=0, \gamma_{R}=\pi$ (OD) and $\gamma_{T}=\gamma_{R}=0$ (SD). Both the $\mathrm{Tx}$ and $\mathrm{Rx}$ were equipped with one omnidirectional antenna, i.e., SISO case. Based on the measured scenarios in [1], we choose the environment-related parameters for OD and SD scenarios as shown in Table II. The Ricean factor and energyrelated parameters shown in Table II for OD and SD scenarios are chosen by considering the constraints of them for different taps as mentioned in Section II. In order to match the measured Doppler PSDs reported in [1] for OD-LVTD and SD-LVTD scenarios, the influence of the LoS component is removed in Figs. 4 and 5 as addressed in [1]. The excellent agreement between the theoretical and measured Doppler PSDs confirms the utility of the proposed wideband model. Unfortunately, to the best of the authors' knowledge, no measurement results (e.g., in [1][2]) were available regarding the impact of the HVTD (e.g., a traffic jam) on the Doppler PSD. Comparing the Doppler PSDs with different VTDs in Figs. 4 and 5, we observe that the V2V channels show highly dynamic Doppler spectrum and the VTD significantly affects the Doppler PSDs at different taps in V2V channels. Figs. 4 and 5 also show that V2V channels have largely spread Doppler PSD and the

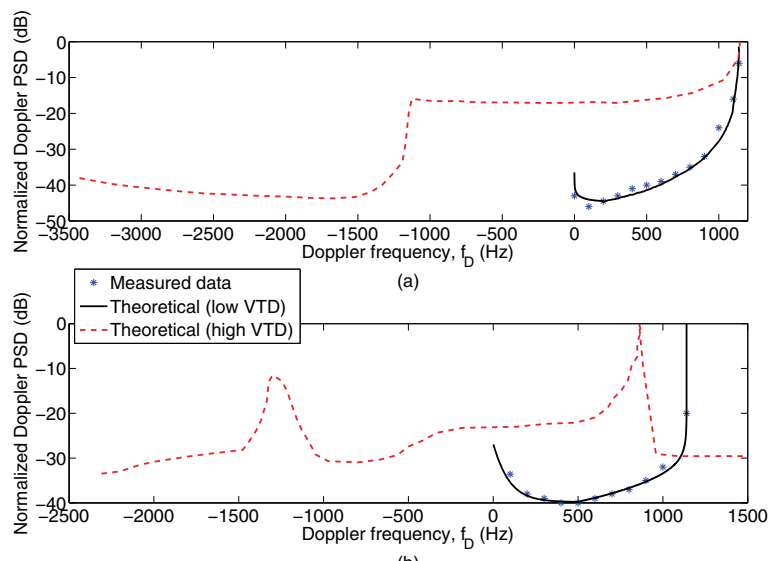

(b)

Fig. 4. Normalized Doppler PSDs of the (a) first tap and (b) second tap of the proposed wideband MIMO V2V channel model with low and high VTDs when the Tx and Rx move in opposite directions on an expressway.

V2V channel with the HVTD has larger spread and more distributed Doppler PSD compared with the V2V channels with the LVTD.

In total, 4 different $\mathrm{V} 2 \mathrm{~V}$ channels are used in this section, i.e., SD-LVTD V2V channel, SD-HVTD V2V channel, ODLVTD V2V channel, and OD-HVTD V2V channel. By comparing the Doppler PSDs in Figs. 4 and 5 with those in Fig. 2 in [10], it is obvious that the Doppler PSD obtained from the proposed model in this paper expresses higher dynamic 


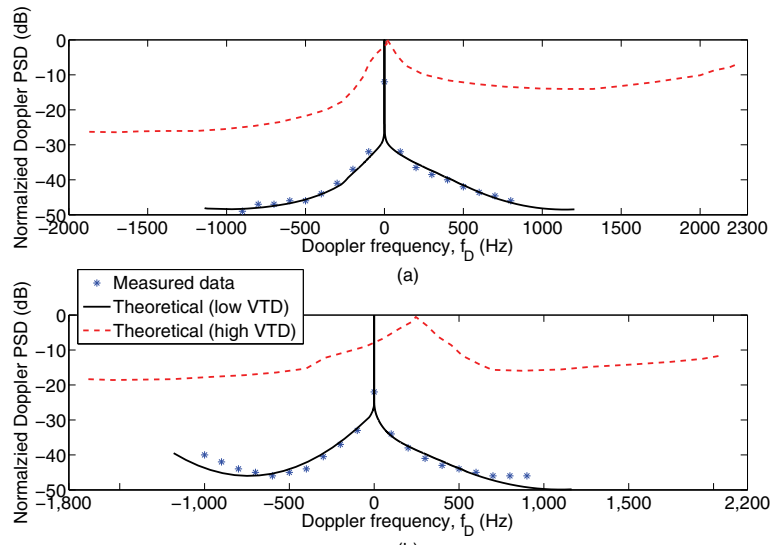

(b)

Fig. 5. Normalized Doppler PSDs of the (a) first tap and (b) second tap of the proposed wideband MIMO V2V channel model with low and high VTDs when the Tx and Rx move in the same direction on an expressway.

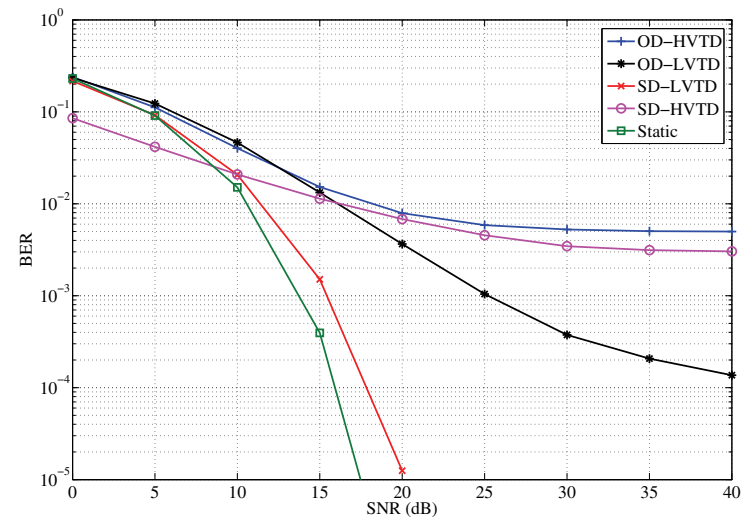

Fig. 6. BER performance of the real V2V system based on IEEE 802.11p standard for different channels.

Doppler spectrum. This indicates that the simple and unrealistic assumption widely used in [7]-[10] that assumes the same Doppler frequency for both moving and static scatterers actually under-estimates the dynamics of the Doppler spectrum of real V2V channels.

Fig. 6 shows the BER performance of the real V2V system without considering pilot symbols for these $4 \mathrm{~V} 2 \mathrm{~V}$ channels. Moreover, the BER performance of the static channel whose parameters are the same as those of the SD-LVTD V2V channel with $v_{T}=v_{R}=v_{S T_{1(2)}}=v_{S R_{1(2)}}=0$ is also illustrated for a reference. As expected, Fig. 6 illustrates that compared with the static channel, V2V channels significantly reduce the performance of the real $\mathrm{V} 2 \mathrm{~V}$ system due to the largely spread Doppler caused by fast moving environments. In general, the OD V2V channel has higher Doppler spectrum than the SD one and the HVTD V2V channel expresses higher Doppler spectrum than the LVTD one. Therefore, the BER for the SDLVTD V2V channel is the best one, while the BER for the OD-HVTD V2V channel is the worst one as shown in Fig. 6.

Fig. 7 shows the BER performance of the real V2V system without considering pilot symbols for OD-LVTD and ODHVTD channels when either ICI self-cancellation schemes or PBC schemes are applied. The symbols in all above cases

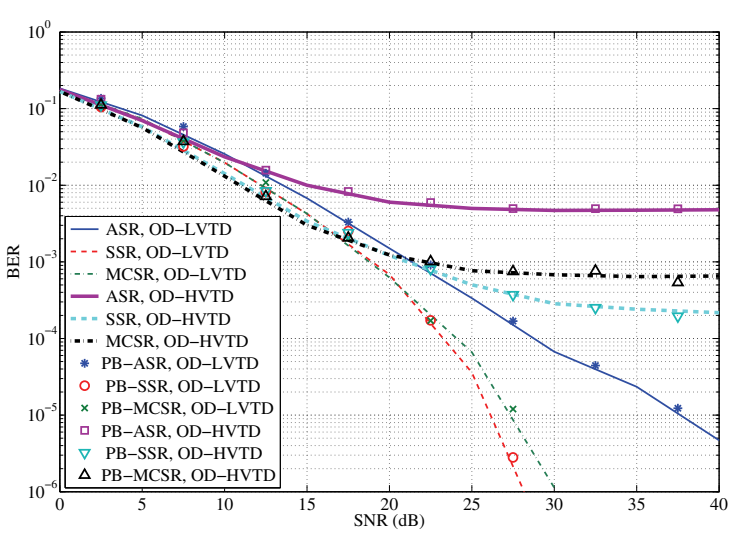

Fig. 7. BER performance of different ICI self-cancellation schemes and PBC schemes in a real V2V system without considering pilots for OD-LVTD and OD-HVTD V2V channels.

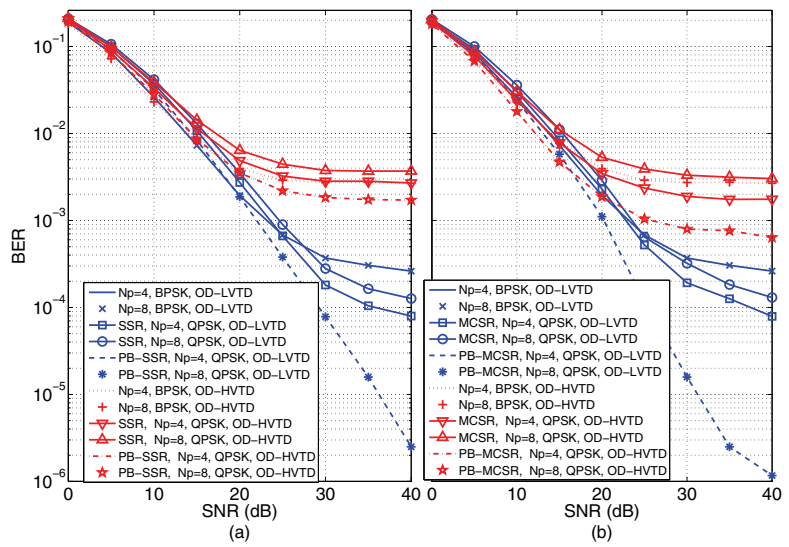

Fig. 8. BER performance of (a) SSR ICI self-cancellation scheme and PB-SSR scheme and (b) MCSR ICI self-cancellation scheme and PB-MCSR scheme in a real V2V system with different number of pilots for OD-LVTD and OD-HVTD V2V channels.

are drawn from QPSK constellation. As expected, the BER performance of ICI self-cancellation schemes is similar to the one of PBC schemes, which validates the proof in Appendix C. Moreover, it can be seen from Fig. 7 that the SSR and MCSR outperform the ASR. This is because that the larger frequency separation in the subcarrier mapping criterion for the SSR and MCSR helps collect the additional frequency diversity and thus enhances their capability in the ICI cancellation.

Fig. 8 shows the performance of the implementation of both ICI self-cancellation schemes and PBC schemes into real V2V systems for OD-LVTD and OD-HVTD channels. The real V2V system without using any ICI cancellation schemes is also considered for a comparison purpose in Fig. 8. To guarantee the same spectral efficiency, we use BPSK modulation for the V2V system without using ICI cancellation schemes and the QPSK modulation for the V2V system using ICI cancellation schemes. To show the impact of pilot symbols, equally-spaced pilot symbols with the number $N p=4$ and $N p=8$ are considered. From Fig. 8, it is clear that the presence of pilot symbols significantly deteriorates the performance of ICI self-cancellation schemes since the pilot symbols will break the mapping criterion as explained in 


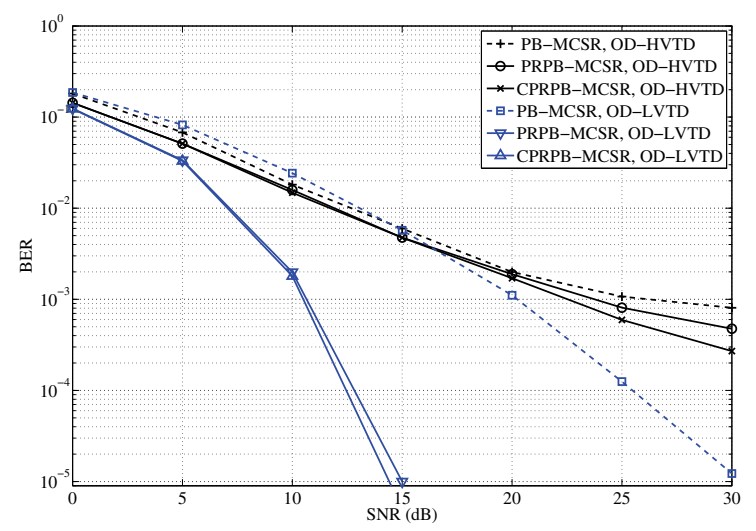

Fig. 9. BER performance of PB-MCSR, PRPB-MCSR, and CPRPB-MCSR schemes in a real V2V system for OD-LVTD and OD-HVTD V2V channels.

Section III. Moreover, the performance degradation increases with the increase of the number of pilot symbols. While the PBC schemes show unchanged performance for different number of pilot symbols and better performance than ICI selfcancellation schemes. Note that compared with IEEE 802.11p standard with 4 pilot symbols in one OFDM block, future $\mathrm{V} 2 \mathrm{~V}$ systems need to add more pilot symbols in order to get more accurate channel estimation. In this case, the advantage of the proposed PBC schemes for real V2V systems is more obvious.

Fig. 9 illustrates the BER performance of the real V2V system with the PB-MCSR, PRPB-MCSR, and CPRPB-MCSR schemes for OD-LVTD and OD-HVTD channels. For the PRPB-MCSR, the phase rotation is obtained by using the estimated normalized frequency offset $\hat{\varepsilon}$ from the preamble. While for the CPRPB-MCSR, the constant phase is obtained based on the normalized frequency offset range $[\hat{\varepsilon}-\varpi, \hat{\varepsilon}+\varpi]$ with $\varpi=0.02$ in this simulation. As shown in Fig. 9, the CPRPB-MCSR expresses the best performance, validating the better performance of the CPRA method over the PRA one for real V2V systems. Note that the performance gap between the CPRA and PRA methods becomes wider when the V2V system has better OFDM spectral efficiency, i.e., more subcarriers in one OFDM block. This is because that in this case, the weight of Doppler spectrum in the generation of frequency offset becomes larger. Based on the results in Figs. 8 and 9, it allows us to conclude that the proposed PBC schemes with the proposed CPRA method are the best ICI cancellation schemes for real V2V systems.

\section{Conclusions}

This paper has proposed a new wideband MIMO V2V RSGBSM, which has the ability to practically investigate the Doppler spectrum and the impact of the VTD on channel statistics. From the proposed model, the Doppler PSD has been derived and analyzed. Numerical results have demonstrated that the VTD has a great impact on the Doppler PSD. Also, the Doppler PSD obtained from our model closely matches the measured data, which validates the utility of the proposed model. To combat the ICI caused by highly dynamic Doppler spectrum in real V2V systems, a new type of ICI cancellation scheme has been proposed combining the advantages of both ICI self-cancelaltion and two-path cancellation schemes. Therefore, the proposed PBC scheme has lower implementation complexity than the two-path cancellation scheme and better performance than the ICI self-cancellation scheme for real V2V systems. To further improve the PBC scheme, we have proposed a new PRA method. Numerical results have shown that compared with the existing PRA method, the proposed CPRA method has better performance and significantly less implementation complexity.

\section{APPENDIX}

A. Derivation of the Condition $\max \left\{R_{T}, R_{R}\right\}<$ $\operatorname{MIN}\left\{a_{l}-a_{l-1}\right\}$ THAT GUARANTEES THE TDL STRUCTURE OF OUR MODEL

For the first tap, Fig. 2 clearly shows that the longest distance caused by the double-bounced rays is the link $O_{T}-A-B-O_{R}$, which is equal to $2 R_{T}+2 R_{R}+2 f$. According to the TDL structure, the inequality $2 R_{T}+2 R_{R}+2 f<2 a_{2}$ should be fulfilled. Considering $R_{T}+R_{R}+2 f<2 a_{1}$ (as shown in Fig. 2) and based on the transitivity of inequalities, we know that if $R_{T}+R_{R}+2 a_{1}<2 a_{2}$ then $2 R_{T}+2 R_{R}+2 f<2 a_{2}$. Therefore, we can conclude that the condition $R_{T}+R_{R}<2 a_{2}-2 a_{1}$ guarantees the fulfillment of the TDL structure for the first tap.

For other taps $\left(l^{\prime}>1\right)$, since the derivations of the condition that guarantees the fulfillment of the TDL structure are the same, here we only detail the derivation of the condition for the second tap. From Fig. 2, it is clear that the longest distance in the second tap caused by the double-bounced rays is either the link $O_{T}-C-S-O_{R}$, which is equal to $2 R_{T}+2 a_{2}$, or the link $O_{T}-S-D-O_{R}$, which is equal to $2 R_{R}+2 a_{2}$. In terms of the TDL structure, the inequality $\max \left\{R_{T}, R_{R}\right\}<\left\{a_{2}-a_{1}\right\}$ should be fulfilled. Therefore, we can conclude that the condition $\max \left\{R_{T}, R_{R}\right\}<\min \left\{a_{l^{\prime}}-a_{l 4}\right\}$ guarantees the fulfillment of the TDL structure for other taps.

Since the condition $R_{T}+R_{R}<2 a_{2}-2 a_{1}$ for the first tap can be rewritten as $R_{T}<a_{2}-a_{1}$ (if $R_{T} \geq R_{R}$ ) and $R_{R}<$ $a_{2}-a_{1}$ (if $R_{R} \geq R_{T}$ ), we can obtain the general condition $\max \left\{R_{T}, R_{R}\right\}<\min \left\{a_{l}-a_{l-1}\right\}$ that guarantees the fulfillment of the TDL structure of our model.

\section{B. Derivation of the Angle $\phi_{S}^{\left(n_{1,1} n_{1,3}\right)}$}

Considering the triangle $\left(S_{T}, O_{T}, S_{R}\right)$ and applying the law of sines, we have

$$
\frac{d\left(S_{T}-O_{T}\right)}{\sin \angle O_{T} S_{R} S_{T}}=\frac{d\left(S_{R}-S_{T}\right)}{\sin \angle S_{R} O_{T} S_{T}}
$$

where $d\left(S_{T}-O_{T}\right)=R_{T}$. Applying the law of cosines to the triangle $\left(O_{T}, S_{R}, O_{R}\right)$ with the assumption of $D>R_{R}$ and to the triangle $\left(S_{T}, O_{T}, S_{R}\right)$, we have $d\left(O_{T}-S_{R}\right)=\xi_{T} \approx$ $D+R_{R} \cos \phi_{R}^{\left(n_{1,3(4)}\right)}$ and $d\left(S_{T}-S_{R}\right)=d_{1}=\left[R_{T}^{2}+\xi_{T}^{2}-\right.$ $\left.2 R_{T} \xi_{T} \cos \angle S_{R} O_{T} S_{T}\right]^{1 / 2}$, respectively. Based on the basic geometry knowledge, we have $\angle O_{R} O_{T} S_{R}=\angle A_{R} S_{R} O_{T}=$ $2 \pi-\phi_{T}^{\left(n_{1,3}\right)}$ and $\angle A_{R} S_{R} R_{T}=\angle S_{R} S_{T} B_{R}=\phi_{S}^{\left(n_{1,1} n_{1,3}\right)}$. Therefore, we can obtain $\angle O_{T} S_{R} S_{T}=\angle A_{R} S_{R} S_{T}-$ $\angle A_{R} S_{R} O_{T}=\phi_{S}^{\left(n_{1,1} n_{1,3}\right)}-2 \pi+\phi_{T}^{\left(n_{1,3}\right)}$ and $\angle S_{R} O_{T} S_{T}=$ $\angle S_{R} O_{T} O_{R}+\angle S_{T} O_{T} O_{R}=2 \pi-\phi_{T}^{\left(n_{1,3}\right)}+\phi_{T}^{\left(n_{1,1}\right)}$. 


\section{Proof of the Same ICI Mitigation Performance between $P B C$ Schemes and ICI Self-Cancellation Schemes}

Since the proof for different mapping criteria and mapping operations is similar, this appendix details the proof for the mirror mapping criterion with conjugate operation, while others are omitted here for brevity. From (14), the combination of received signals at the $m$-th subcarrier for the PB-MCSR is $\widehat{X}_{m}=\frac{H_{m, m}^{*} \bar{Y}_{m}^{(1)}+H_{-m,-m}^{*} \bar{Y}_{N-m}^{(2)}}{\left|H_{m, m}\right|^{2}+\left|H_{-m,-m}\right|^{2}}$, which can be further simplified as

$\widehat{X}_{m}=X_{m}+\frac{\sum_{k=0, k \neq m}^{N-1}\left(H_{m, m}^{*} H_{m, k}+H_{-m,-m} H_{-m,-k}^{*}\right) X_{k}^{*}}{\left|H_{m, m}\right|^{2}+\left|H_{-m,-m}\right|^{2}}$.

Comparing the expression of the $m$-th symbol $\widehat{X}_{m}$ for the MCSR in (14) and (21), it can be readily verified that they share the same CIR as

$$
\begin{aligned}
& C I R_{M C S R}=C I R_{P B-M C S R}= \\
& \frac{E\left\{\left|H_{m, m}\right|^{2}+\left|H_{-m,-m}\right|^{2}\right\}}{\sum_{k=0, k \neq m}^{N-1} E\left\{\left|H_{m, m}^{*} H_{m, k}+H_{-m,-m} H_{-m,-k}^{*}\right|^{2}\right\}} .
\end{aligned}
$$

Therefore, the MCSR and PB-MCSR have the same ICI mitigation performance.

\section{REFERENCES}

[1] G. Acosta and M. A. Ingram, "Six time- and frequency-selective empirical channel models for vehicular wireless LANs," IEEE Veh. Technol. Mag., vol. 2, no. 4, pp. 4-11, Dec. 2007.

[2] I. Sen and D. W. Matolak, "Vehicle-vehicle channel models for the 5GHz band," IEEE Trans. Intell. Transp. Syst., vol. 9, no. 2, pp. 235-245, June 2008.

[3] C.-X. Wang, X. Cheng, and D. I. Laurenson, "Vehicle-to-vehicle channel modeling and measurements: Recent advances and future challenges," IEEE Commun. Mag., vol. 47, no. 11, pp. 96-103, Nov. 2009.

[4] J. Maurer, T. Fügen, M. Porebska, T. Zwick, and W. Wisebeck, "A rayoptical channel model for mobile to mobile communications," COST 2100 4th MCM, COST 2100 TD(08) 430, Feb. 2008.

[5] A. S. Akki and F. Haber, "A statistical model for mobile-to-mobile land communication channel," IEEE Trans. Veh. Technol., vol. 35, no. 1, pp. 2-10, Feb. 1986.

[6] A. G. Zajić and G. L. Stüber, "Space-time correlated mobile-to-mobile channels: Modelling and simulation," IEEE Trans. Veh. Technol., vol. 57, no. 2, pp. 715-726, Mar. 2008

[7] X. Cheng, C.-X. Wang, B. Ai, and H. Aggoune, "Envelope level crossing rate and average fade duration of non-isotropic vehicle-tovehicle Ricean fading channels," IEEE Trans. Intell. Transp. Syst., accepted for publication.

[8] X. Cheng, C.-X. Wang, D. I. Laurenson, S. Salous, and A. V. Vasilakos, "An adaptive geometry-based stochastic model for non-isotropic MIMO mobile-to-mobile channels," IEEE Trans. Wireless Commun., vol. 8, no. 9, pp. 4824-4835, Sep. 2009.

[9] X. Cheng, C.-X. Wang, and D. I. Laurenson, "A novel three-dimensional regular-shaped geometry-based stochastic model for MIMO mobile-tomobile Ricean fading channels," in Proc. IEEE VTC-Fall, Sep. 2010, pp. 1-5.

[10] X. Cheng, C.-X. Wang, and D. I. Laurenson, H. H. Chen, and A. V. Vasilakos, "A geometry-based stochastic model for wideband MIMO mobile-to-mobile channels," in Proc. IEEE Globecom, Nov. 2009, pp. 16.

[11] A. G. Zajić, G. L. Stüber, T. G. Pratt, and S. Nguyen, "Wideband MIMO mobile-to-mobile channels: Geometry-based statistical modeling with experimental verification," IEEE Trans. Veh. Technol., vol. 58, no. 2, pp. 517-534, Feb. 2009.
[12] J. Karedal, F. Tufvesson, N. Czink, A. Paier, C. Dumard, T. Zemen, C. F. Mecklenbräuker, and A. F. Molisch, "A geometry-based stochastic MIMO model for vehicle-to-vehicle communications," IEEE Trans. Wireless Commun., vol. 8, no. 7, pp. 3646-3657, July 2009.

[13] IEEE P802.11p-2010: Part 11: Wireless LAN Medium Access Control (MAC) and Physical Layer (PHY) Specifications: Amendment 6: Wireless Access in Vehicular Environments, DOI: 10.1109/IEEESTD.2010. 5514475, July 15, 2010.

[14] J. Ahn and H. S. Lee, "Frequency domain equalization of OFDM signal over frequency nonselective Rayleigh fading channels," Electron. Lett., vol. 29, no. 16, pp. 1476-1477, Aug. 1993.

[15] S. H. Müller-Weinfurtner, "Optimum Nyquist windowing in OFDM receivers," IEEE Trans. Commun., vol. 49, no. 3, pp. 417-420, Mar. 2001.

[16] Y. Zhao, J. D. Leclercq, and S. Häggman, "Intercarrier interference compression in OFDM communication systems by using correlative coding," IEEE Commun. Lett., vol. 2, no. 8, pp. 214-216, Aug. 1998.

[17] Y. Zhao and S. G. Häggman, "Intercarrier interference self-cancellation scheme for OFDM mobile communication systems," IEEE Trans. Commun., vol. 49, no. 7, pp. 1185-1191, July 2001.

[18] L. Song, R. Lamare, and A. G. Burr, "Successive interference cancellation schemes for time-reversal space-time block codes," IEEE Trans. Veh. Technol., vol. 57, no. 1, pp. 1-7, Jan. 2008.

[19] Y. Fu, S. G. Kang, and C. C. Ko, "A new scheme for PAPR reduction in OFDM systems with ICI self-cancellation," in Proc. IEEE VTC-Fall, Sep. 2002, pp. 1418-1421.

[20] K. Sathananthan, R. M. A. P. Rajatheva, and S. B. Slimane, "Cancellation technique to reduce intercarrier interference in OFDM," Electron. Lett., vol. 36, no. 25, pp. 2078-2079, Dec. 2000.

[21] K. Sathananthan, C. R. N. Athandage, and B. Qin, "A novel ICI cancellation scheme to reduce both frequency offset and IQ imbalance effects in OFDM," in Proc. IEEE ISCC, July 2004, pp. 708-713.

[22] M. Wen, X. Cheng, X. Wei, B. Ai, and B. Jiao, "A novel effective ICI self-cancellation method," in Proc. IEEE Globecom, Dec. 2011.

[23] H. G. Yeh and C. C. Wang, "New parallel algorithm for mitigating the frequency offset of OFDM systems," in Proc. IEEE VTC-Fall, Sep. 2004, pp. 2087-2091.

[24] H. G. Yeh, Y. K. Chang, and B. Hassibi, "A scheme for cancelling intercarrier interference using conjugate transmission in multicarrier communication systems," IEEE Trans. Wireless Commun., vol. 6, no. 1, pp. 3-7, Jan. 2007.

[25] C. L. Wang and Y. C. Huang, "Intercarrier interference cancelling using general phase rotated conjugate transmission for OFDM systems," IEEE Trans. Commun., vol. 58, no. 3, pp. 812-819, Mar. 2010.

[26] M. Wen, X. Cheng, L. Yang, and B. Jiao, "Two-path transmission framework for ICI reduction in OFDM systems," in Proc. IEEE Globecom, Dec. 2013, submitted for publication.

[27] C.-X. Wang, D. Yuan, H.-H. Chen, and W. Xu, "An improved deterministic SoS channel simulator for efficient simulation of multiple uncorrelated Rayleigh fading channels," IEEE Trans. Wireless Commun., vol. 7, no. 9, pp. 3307-3311, Sep. 2008.

[28] C.-X. Wang, M. Pätzold, and D. Yuan, "Accurate and efficient simulation of multiple uncorrelated Rayleigh fading waveforms," IEEE Trans. Wireless Commun., vol. 6, no. 3, pp. 833-839, Mar. 2007.

[29] M. Pätzold, C.-X. Wang, and B. O. Hogstad, "Two new sum-ofsinusoids-based methods for the efficient generation of multiple uncorrelated Rayleigh fading waveforms," IEEE Trans. Wireless Commun., vol. 8, no. 6, pp. 3122-3131, June 2009.

[30] C.-X. Wang, X. Hong, H. Wu, and W. Xu, "Spatial temporal correlation properties of the 3GPP spatial channel model and the Kronecker MIMO channel model," EURASIP J. Wireless Commun. Netw., vol. 2007, article ID 39871, 2007.

[31] X. Cheng, C.-X. Wang, H. Wang, X. Gao, X.-H. You, D. Yuan, B. Ai, Q. Huo, L. Song, and B. Jiao, "Cooperative MIMO channel modeling and multi-link spatial correlation properties," IEEE J. Sel. Areas Commun., vol. 30, no. 2, pp. 388-396, Feb. 2012.

[32] X. Cheng, C.-X. Wang, D. I. Laurenson, S. Salous, and A. V. Vasilakos, "New deterministic and stochastic simulation models for non-isotropic scattering mobile-to-mobile Rayleigh fading channels," Wireless Commun. Mobile Comput., John Wiley \& Sons, vol. 11, no. 7, pp. 829-842, July 2011.

[33] I. S. Gradshteyn and I. M. Ryzhik, Table of Integrals, Series, and Products, 6th ed. Boston: Academic, 2000. 


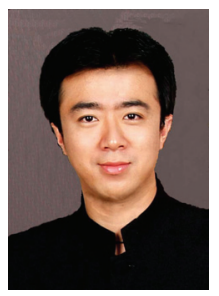

Xiang Cheng (S'05-M'10-SM'13) received the $\mathrm{Ph} . \mathrm{D}$. degree from Heriot-Watt University and the University of Edinburgh, Edinburgh, UK, in 2009, where he received the Postgraduate Research Thesis Prize.

He has been with Peking University, Bejing, China, since 2010, first as a Lecturer, and then as an Associate Professor since 2012. His current research interests include mobile propagation channel modeling and simulation, next generation mobile cellular systems, intelligent transport systems, and hardware prototype development and practical experiments.

$\mathrm{He}$ has published more than 60 research papers in journals and conference proceedings. He received the best paper award from the IEEE International Conference on ITS Telecommunications (ITST 2012). Dr. Cheng received the "2009 Chinese National Award for Outstanding Overseas Ph.D. Student" for his academic excellence and outstanding performance. He has served as Symposium Co-Chair and a Member of the Technical Program Committee for several international conferences.

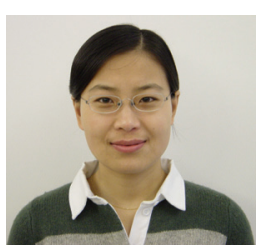

Qi Yao received the B.Sc. and M.Eng. degrees in communication and information systems from Shandong University, China, in 1997 and 2000, respectively.

She has been with Heriot-Watt University, Edinburgh, UK, since 2007, where she is now working as a Research Associate. She was a Research Fellow at the University of Agder, Grimstad, Norway, from 2001-2006, and a Research Assistant at the Technical University of Hamburg-Harburg, Hamburg, Germany, from 2000-2001. Her current research interests include wireless communications, wireless channel modeling and simulation, and MIMO. She has published over 40 papers in refereed journals and conference proceedings.

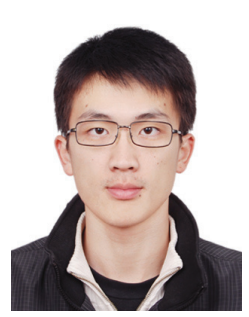

Miaowen Wen received the B.Eng. degree in information engineering from Beijing Jiaotong University, Beijing, China, in 2009. He is currently pursuing his Ph.D. degree at Peking University, Beijing, China. Since September 2012, he has been a visiting student research collaborator at Princeton University, Princeton, USA. His recent research interests include multiple access techniques, MIMO, and cooperative communication.

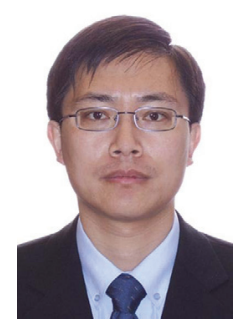

Cheng-Xiang Wang (S'01-M'05-SM'08) received the B.Sc. and M.Eng. degrees in communication and information systems from Shandong University, China, in 1997 and 2000, respectively, and the Ph.D. degree in wireless communications from Aalborg University, Denmark, in 2004.

He has been with Heriot-Watt University, Edinburgh, UK, since 2005, first as a Lecturer, then as a Reader in 2009, and was promoted to Professor in 2011. He is also an Honorary Fellow of the University of Edinburgh, UK, and a Chair/Guest Professor of Shandong University, Huazhong University of Science and Technology, and Southeast University, China. He was a Research Fellow at the University of Agder, Grimstad, Norway, from 2001-2005, a Visiting Researcher at Siemens AG-Mobile Phones, Munich, Germany, in 2004, and a Research Assistant at the Technical University of Hamburg-Harburg, Hamburg, Germany, from 2000-2001. His current research interests include wireless channel modeling and simulation, green communications, cognitive radio networks, vehicular communication networks, large MIMO, cooperative MIMO, and B4G wireless communications. He has published one book chapter and more than 180 papers in refereed journals and conference proceedings.

Prof. Wang served or is currently serving as an editor for eight international journals, including the IEEE TRANSACTIONS ON VEHICULAR TECHNOLOGY (2011-) and the IEEE TRANSACTIONS ON WIRELESS COMMUNICATIONS (2007-2009). He was the leading Guest Editor for the IEEE JOURNAL on Selected Areas in Communications, Special Issue on Vehicular Communications and Networks. He served or is serving as a TPC member, TPC Chair, and General Chair for over 70 international conferences. He received Best Paper Awards from IEEE Globecom 2010, IEEE ICCT 2011, and ITST 2012. He is a Fellow of the IET, a Fellow of the HEA, and a member of the EPSRC Peer Review College.

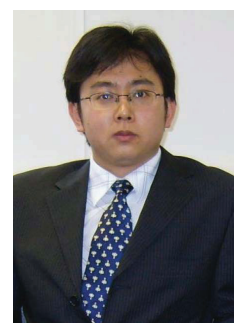

Ling-Yang Song (S'03-M'06-SM'12) received a $\mathrm{Ph} . \mathrm{D}$. from the University of York, UK, in 2007, where he received the K. M. Stott Prize for excellent research. He worked as a postdoctoral research fellow at the University of Oslo, Norway, and Harvard University, until rejoining Philips Research UK in March 2008. In May 2009, he joined the School of Electronics Engineering and Computer Science, Peking University, China, as a full professor. His main research interests include MIMO, OFDM, cooperative communications, cognitive radio, physical layer security, game theory, and wireless ad hoc/sensor networks.

$\mathrm{He}$ is co-inventor of a number of patents (standard contributions), and author or co-author of over 100 journal and conference papers. He received the best paper award at the IEEE International Conference on Wireless Communications, Networking and Mobile Computing (WiCOM 2007), the best paper award at the 1st IEEE International Conference on Communications in China (ICCC 2012), the best student paper award at the 7th International Conference on Communications and Networking in China (ChinaCom2012), the best paper award at the IEEE Wireless Communication and Networking Conference (WCNC2012), and the best paper award at the International Conference on Wireless Communications and Signal Processing (WCSP 2012).

$\mathrm{He}$ is currently on the Editorial Board of IET Communications and the Journal of Network and Computer Applications, and a guest editor of Elsevier Computer Communications and the EURASIP Journal on Wireless Communications and Networking. He serves as a member of the Technical Program Committee and Co-chair for several international conferences and workshops.

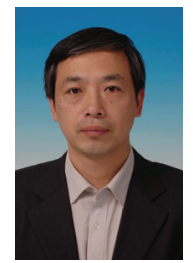

Bingli Jiao received B.S. and M.S. degrees from Peking University, China in 1983 and 1988, respectively, and received a Ph.D. from the University of Saaland, F. R., Germany in 1995. He became an associate professor and professor with Peking University in 1995 and 2000, respectively. His current interests include communication theory and techniques and sensor design. 